\title{
On the Epinephrine in the Blood of the Suprarenal Vein and in the Suprarenal Extract from the Rabbit during Pregnancy.
}

\author{
By \\ TETSUTARO AOMURA. \\ (青朴瀻火郎) \\ (From the Physiological Laboratory of Prof. I. Satalie', \\ Toholu Imperial University, Sendai.)
}

In the course of my previous investigations, ${ }^{1)}$ comparing the epinephrine values obtainable by the cat paradoxical eye reaction and those obtainable by the rabbit intestine strip method of the suprarenal vein blood (the cava pocket specimen) of some species of animals, I became aware by chance that a pregnant rabbit yielded in this relation some different features, that is to say, the ratio of the two values is the reverse of those in the normal, i. e., non-pregnant rabbit. For the non-pregnant rabbit the epinephrine value obtainable by the paradoxical eye reaction is invariably larger than that by the rabbit intestine, the a verage of the ratio of both values being found as (Eye : Intestine) $2.2: 1$. In this pregnant animal the rabbit intestine procedure yielded rather a greater value than the denervated cat pupil. It must be here mentioned that these estimations are carried out with adrenalin chloride preparation of the Sankyo Co. as the standard.

At first we suspected that the rosult might be taken as an exception or as due to some unwarranted terhnical error, but soon we came across another instance, which gave us a similar result as the first case, then we undertook a systematic investigation of the suprarenal vein blood of the rabbit in respect of the action upon both these kinds of tissues through the whole pregnant stage, the results of which form the content of the present paper.

1) T. Aomura, Tohoku J. Exp. Med., 1929, 14, 291. 


\section{Methods :}

The rabbits employed in this investigation were copulated by myself or Mr. Miy ake, Operation assistant of this Laboratory. The date of the conception was fixed, and on the 4th to the 30th day of pregnancy the animals were experimented on, only Rabbits 32 and 33 being experimented on about 8 hours and 2 days respectively after the delivery, i.e. in the puerperal period. All the rabbits were dissected, and the uterus and its content were carefully observed. The embryos on the 4th day of pregnancy were macroscopically sought out with success.

The suprarenal vein blood was collected by means of the cava pocket prepared without anaesthetising. In the case one and the same cava pocket specimen an attempt was made to estimate the epinephrine in parallel by means of the paradoxical eye reaction of cat, both splanchnic nerves of which and one superior cervical ganglion were previously interfered with, and by the rabbit intestinal segment method, the adrenalin chloride preparation of the Sank yo Co. being the standard.

After termination of the cava pocket experiment both suprarenal capsules were removed and, extraction being performed by means of the procedure of Folin, Cannon and Denis. Both the biological methods above mentioned and the modification by Koda ma of Folin's and other's colorimetric method were used for estimation of epinephrine for one and the same sample of extract. It should be understood that all the methods and procedures here employed were those described in the foregoing paper. ${ }^{1)}$

\section{Results:}

46 pregnant rabbits and 2 rabbits in the puerperal stadium were employed. The reversal ratio against the normal, viz. non-pregnant rabbit of the epinephrine values obtainable by both methods were detected from 21 st to the 29th days of pregnancy, with definiteness especially from the 22nd to the 28th day. But a few exceptional cases were also noted, there a large number of rabbits in these days of pregnancy was brought to the test of experiment. The data on normal rabbits involved in the previous communication ${ }^{13}$ serve as control for the present results.

In the first place the results on the suprarenal vein blood, which are supplied in Table I, will be discussed, and then those on the gland extract in the second place. One example will be given in full to illustrate the experimental course in general. 


\section{(1) Epinephrine in the Suprarenal Vein Blood in Pregnant Rabbits.}

As is well known, the pregnancy of rabbits is completed in most cases in 30 days after conception; the earliest stage of pregnancy investigated by us was 4th day and the examination was followed up not only to the last day of pregnancy, but a few days after the delivery.

The experimental results are summarized in the order of days of the preguancy. At first the ratio of the values obtained by both biological methods will be discussed. In the non-pregnant rabbits, reported in the foregoing paper, it was $2.2: 1$ on an average (from $1.2: 1$ to $4: 1$ ). It was now calculated for 6 rabbits in the 4 th to 10 th day of pregnancy as $2.3: 1$ to $1.3: 1$, mean $1.7: 1$, the superiority of the value obtainable by the paradoxical eye reaction against that by the rabbit intestine showed already a tendency to decrease. The ratio further gradually inclined to become smaller for 9 rabbits with advance of the pregnancy, that is, on the 12th to 20 th days it was computed as from $2.0: 1$ to $1.2: 1$, on an average $1.4: 1$ (from 12 pairs of the determinations). On the 21 st day of the pregnancy (two rabbits, two determinations) the ratio was found as $1: 1$, that is the values obtained by means of both methods coincided with each other. Hence forth the values obtained by the rabbit intestine strip begin to become larger than those by the denervated eye. The ratio yielded from 15 rabbits on the 22 nd to 25 th day of the pregnancy the ratio was $1: 1.1$ to $1: 2.0$, mean

Example.

11. VII. 1928. Rabbit 9. 3.51 kilos. 24 th day of pregnancy.

\begin{tabular}{|c|c|c|c|c|c|}
\hline Time & 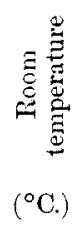 & 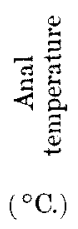 & 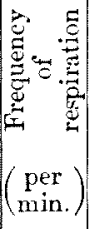 & 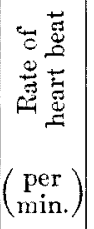 & Experimental processes \\
\hline $\begin{array}{l}9: 18 \text { a.m. } \\
9: 22-9: 58\end{array}$ & 20.5 & 38.5 & 78 & 264 & $\begin{array}{l}\text { Fistend. } \\
\text { Made cava pocket, a. coeliaca and a, mes. sup. } \\
\text { untied. }\end{array}$ \\
\hline $\begin{array}{l}10: 02 \\
10: 11\end{array}$ & 21.0 & 35.3 & 126 & 282 & $\begin{array}{l}\text { Collected cava pocket specimen I, after discard- } \\
\text { ing } 1 \text { c.c. of cava blood. }\end{array}$ \\
\hline $\begin{array}{l}10: 15 \\
10: 30\end{array}$ & 21.5 & 38.0 & 132 & 276 & $\begin{array}{l}\text { Collected cara pocket specimen II, after } \\
\text { discarding I c.c. of cava blood. }\end{array}$ \\
\hline $\begin{array}{l}10: 35 \\
10: 40\end{array}$ & 21.5 & 37.3 & 126 & 270 & Final bleeding from abd. aort. \\
\hline
\end{tabular}


Assays with denervated eye on cat.

20. VI. 1928, 2.59 kilos. Cut both splanchnici extruperitoneally.

4. VII. 1928, 2.20 kilos. Removed left superior cervical ganglion.

11. VII. 1928, 2.00 kilos. Experimented on as follows :

1:00 p.m. 3.0 grms. urethane (1.5 grms. per kilo) by stomach tube.

1:48, Fastend.

1:53 " Put cannula into trachea and external jugular vein.

\begin{tabular}{|c|c|c|c|c|c|c|c|c|}
\hline Time & \multicolumn{2}{|c|}{$\begin{array}{l}\text { Quantity of } \\
\text { cara pocket } \\
\text { specimen (c.c.) } \\
\text { and quantity } \\
\text { of adrenaline } \\
\text { hydrochloride } \\
\text { solution (c.c.) } \\
\text { in a given } \\
\text { concentration* }\end{array}$} & (mms.) & 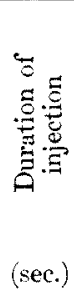 & 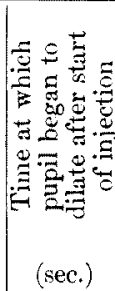 & 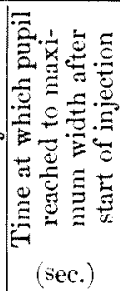 & 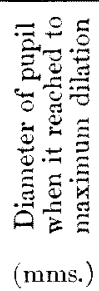 & 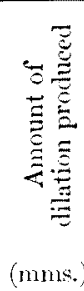 \\
\hline 2:13 p.m. & $1 / 1000$ & 0.1 & 1.5 & 6 & 10 & 15 & 2.0 & 0.5 \\
\hline $2: 20^{1}$ & , & 0.2 & 1.5 & 6 & 10 & 17 & 2.5 & 1.0 \\
\hline $2: 25$ & ," & 0.3 & 1.5 & 6 & 11 & 17 & 8.5 & 2.0 \\
\hline $2: 29$ & 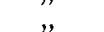 & 0.4 & 1.5 & 6 & 9 & 16 & 4.0 & 2.5 \\
\hline $2: 35$ & & 0.5 & 1.5 & 6 & 9 & $1 \mathrm{~s}$ & $4.5-$ & $3.0-$ \\
\hline $2: 40$ & I & 0.5 & $1.5+$ & 6 & 8 & 15 & 5.0 & 3.5 \\
\hline $2: 45$ & $1 / 1000$ & 0.6 & 1.5 & 6 & 8 & 15 & $5.0+$ & $3.5+$ \\
\hline $2: 50$ & & 0.7 & 1.5 & 6 & 8 & 17 & 5.5 & 4.0 \\
\hline $2 ; 53$ & I & 0.5 & 1.5 & 5 & 8 & 13 & $5.0-$ & 3.5 \\
\hline $2: 58$ & $1 / 1000$ & 0.6 & 1.0 & 6 & 8 & 15 & $4.5+$ & $3.5+$ \\
\hline $3: 04$ & II & 0.5 & 1.0 & 6 & 8 & 16 & 3.5 & 2.5 \\
\hline $3: 09$ & $1 / 1000$ & 0.4 & 1.0 & 6 & 9 & 16 & 3.0 & 2.0 \\
\hline $3: 13$ & II & 0.5 & 1.0 & 6 & 9 & 17 & 3.5 & 2.5 \\
\hline $3: 20$ & $1 / 1000$ & 0.5 & 1.5 & 7 & 10 & 17 & $4.5-$ & 3.0 \\
\hline $3: 26$ & E.R. & 0.5 & 1.5 & 9 & 15 & 24 & 4.0 & 2.5 \\
\hline $3: 30$ & $1 / 1000$ & 0.4 & 1.5 & 9 & 18 & 20 & 3.5 & 2.0 \\
\hline $3: 34$ & & 0.5 & 1.5 & 6 & 9 & 18 & $4.5-$ & $3,0-$ \\
\hline $3: 37$ & E.R. & 0.5 & 1.5 & 7 & 6 & 18 & $4.5-$ & $3,0-$ \\
\hline $3: 40$ & & 0.5 & 1.5 & 6 & 8 & 17 & 4.0 & 2.5 \\
\hline $3: 44$ & $1 / 1000$ & 0.4 & 1.5 & 7 & 9 & 18 & 3.5 & 2.0 \\
\hline $3: 47$ & & 0.5 & 1.5 & 6 & 8 & 17 & 4.5 & 3.0 \\
\hline $3: 50$ & E.R. 2 & 0.5 & $1.5-$ & 6 & 8 & 15 & 4.0 & $2.5+$ \\
\hline $3: 53$ & $1 / 1000$ & 0.4 & 1.5 & 6 & 8 & 16 & 3.5 & 2.0 \\
\hline $8: 57$ & E.L 2 & 0.5 & 1.5 & 6 & 8 & 16 & 4.0 & 2.5 \\
\hline $4: 01$ & $1 / 1000^{\circ}$ & 0.5 & 1.5 & 6 & 9 & 15 & $4.5+$ & $3.0+$ \\
\hline $4: 05$ & E.L. 2 & 0.5 & 2.0 & 6 & 9 & 16 & $4.5+\frac{1}{t}$ & $2.5+$ \\
\hline 4:09 & $1 / 1000$ & 0.4 & 1.5 & 6 & 8 & 16 & 3.5 & 2.0 \\
\hline $4: 15$ & F.L. 2 & 0.5 & 2.0 & 6 & 8 & 16 & $4.5+$ & $2.5+$ \\
\hline $4: 20$ & $1 / 1000$ & 0.5 & 1.5 & 7 & 8 & 16 & $4.5+$ & $3.0+$ \\
\hline
\end{tabular}

* Adrenaline blood solution which was injected for comparison: Ty rode's solution 2.5 $-0.5-2 \alpha$, double Ty rode's solution $\alpha$ c.c., indifferent blood from rabbit which experimented 0.5 c.c., adrenaline hydrochloride solution $\alpha$ c.c. Cava pocket blood solution for injection: c.p. blood 0.5 c.c. and Ty rode's solution 2.0 c.c.

To sum up the results of determination :

Specimen I: Stronger than $0.0005 \mathrm{mgrm}$; weaker than $0.0006 \mathrm{mgrm}$. It was assyed at $0.0011 \mathrm{mgrm}$, in 1 c.c.

Specimen II : Weaker than $0.0006 \mathrm{mgrm}$. and $0.0005 \mathrm{mgrm}$; stronger than 0.0004 mgrm. It was taken as $0.0009 \mathrm{mgrm}$, in 1 c.c.

E.R. $\times 2$ : Weaker than $0.0005 \mathrm{mgrm}$; stronger than $0.0004 \mathrm{mgrm}$. It was assayed 
$0.0009 \mathrm{mgrm}$. in 1 c.c. of diluted solution, i.e. $0.0018 \mathrm{mgrm}$. in 1 c.c. of original solution.

E.L. $\times 2$ : Stronger than $0.0004 \mathrm{mgrm}$; ; weaker than $0.0005 \mathrm{mgrm}$. It was assayed at $0.0009 \mathrm{mgrm}$. in 1 c.c. of diluted solution, i.e. $0.0018 \mathrm{mgrm}$. in 1 c.c. of original solution.

"E.R. $\times 2$ " indicates extract of right adrenal gland which diluted to 2 volumes with aq. dest; " E.L. $\times 2$ " indicates extract of left adrenal gland which diluted to 2 volumes with aq. dest.

Epinephrine concentration and output determined by paradoxical eye reaction :

\begin{tabular}{|c|c|c|c|c|c|c|c|}
\hline \multirow{3}{*}{$\begin{array}{l}\text { No. of cava } \\
\text { pocket } \\
\text { specimen }\end{array}$} & \multicolumn{4}{|c|}{ Blood flow (c.c.) } & \multicolumn{3}{|c|}{$\begin{array}{l}\text { Epinephrine output } \\
\text { (mgrm.) }\end{array}$} \\
\hline & \multirow{2}{*}{ Quantity } & \multirow{2}{*}{$\begin{array}{l}\text { Duration } \\
\text { of } \\
\text { collection } \\
\text { (sec.) }\end{array}$} & \multicolumn{2}{|c|}{$\begin{array}{l}\text { Quantity } \\
\text { per min. }\end{array}$} & \multirow{2}{*}{$\begin{array}{l}\text { Quantity } \\
\text { contained } \\
\text { in } 1 \text { c.c. }\end{array}$} & \multicolumn{2}{|c|}{$\begin{array}{l}\text { Output } \\
\text { per min. }\end{array}$} \\
\hline & & & $\begin{array}{c}\text { per } \\
\text { animal }\end{array}$ & $\begin{array}{l}\text { per } \\
\text { kilo }\end{array}$ & & $\underset{\text { animal }}{\text { per }}$ & $\begin{array}{l}\text { per } \\
\text { kilo }\end{array}$ \\
\hline I & 3.5 & 180 & 1.16 & 0.33 & 0.0011 & 0.0013 & 0.00037 \\
\hline II & 2.6 & 150 & 1.04 & 0.3 & 0.0009 & 0.00094 & 0.00027 \\
\hline
\end{tabular}

Epinephrine content per grm. tissue of the gland determined by paradoxical eye reaction:

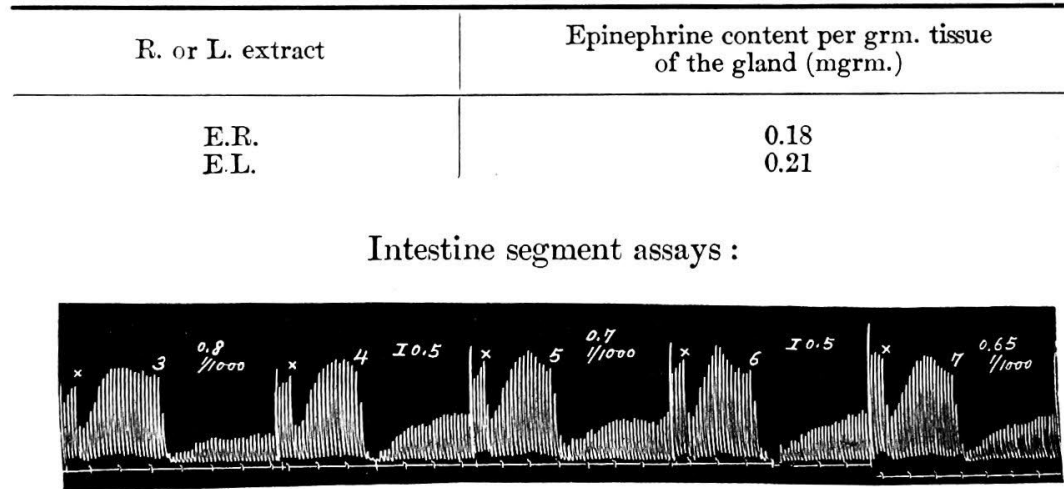

Fig. 1. a (Reduced to $\left.\frac{1}{2}\right)$. I : Decidedly weaker than $0.0008 \mathrm{mgrm}$.; very little weaker than $0.0007 \mathrm{mgrm}$. and almost as strong as $0.00065 \mathrm{mgrm}$.

In all the intestine tracings, at the mark " $\times$ " atropine- $T$ y rod e's solution, in which the rabbit intestine segment was beating rhythmically, was replaced by indifferent blood solution, and at the "numeral" the indifferent blood solution to which a certain quantity of adrenalin chloride of Sankyo Co. was added, or by the cava pocket blood solution.

All the blood solutions were prepared by diluting with 4 volumes of $\mathrm{T}$ y r o d e's solution and the quantity of blood employed for one assay was 0.5 c.c.

The numeral of cava blood specimens and the quantity of adrenalin chloride solution which is showed in c.c. and in concentration were added to each observation. For example, " $0.81 / 1000$ " shows " 0.8 c.c. of adrenalin chloride solution with the concentration of $1 / 1000$ mgrm. in 1 c.c." i.e. 0.0008 mgrm. adrenalin hydrochloride. To show the I cava blood specimen, we used the numeral "I".

In all tracings, time intervals 30 seconds.

Farther see re the procedures of determination: Sugawara, Wat an abé and Saito, Tohoku, j. Exp. Med., 1926, 7, 15. 


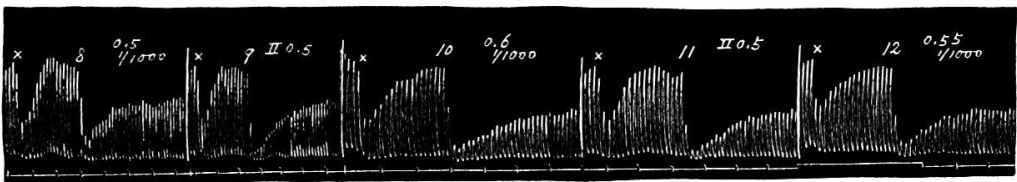

Fig. 1. $b$ (Reduced to $\frac{1}{2}$ ). II : Stronger than $0.0005 \mathrm{mgrm}$; weaker than $0.0006 \mathrm{mgrm}$. and almost as strong as $0.00055 \mathrm{mgrm}$.

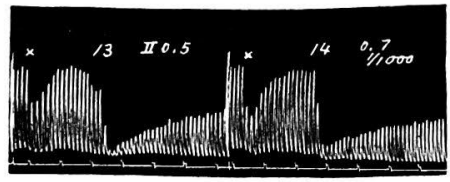

Fig. 1. c (Reduced to $\left.\frac{1}{2}\right)$. II : Weaker than 0.0007 mgrm.

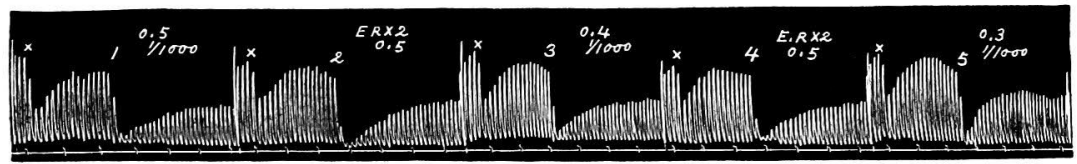

Fig. 1. $\mathrm{d}$ (Reduced to $\frac{1}{2}$ ). E.R. $\times 2$ : Weaker than $0.0005 \mathrm{mgrm}$. ; stronger than 0.0003 mgrm. and not much different from $0.0004 \mathrm{mgrm}$.

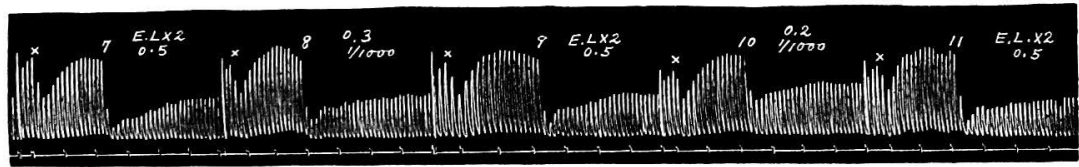

Fig. 1. e (Reduced to $\frac{1}{2}$ ). E.L. $\times 2$ : Stronger than $0.0002 \mathrm{mgrm}$. and $0.00025 \mathrm{mgrm}$., (Obs. 12 with Obs. $11 \& 13$ ) almost as strong as $0.0003 \mathrm{mgrm}$.

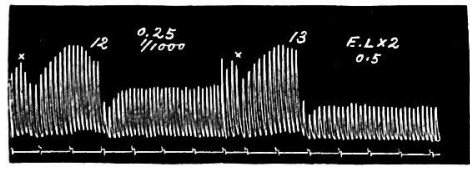

Fig. 1. $\mathbf{f}\left(\right.$ Reduced to $\left.\frac{1}{2}\right)$. E.L. $\times 2:$ Stronger than $0.00025 \mathrm{mgrm}$.

To sum up :

Specimen I: Decidedly weaker than $0.0008 \mathrm{mgrm}$; very little weaker than 0.0007 mgrm. and almost as strong as $0.00065 \mathrm{mgrm}$. It was assayed at $0.0013 \mathrm{mgrm}$. in 1 c.c.

Specimen II : Stronger than $0.0005 \mathrm{mgrm}$. ; weaker than $0.0006 \mathrm{mgrm}$. and almost as strong as $0.00055 \mathrm{mgrm}$. It was taken as $0.0011 \mathrm{mgrm}$. in 1 c.c.

E.R. $\times 2$ : Weaker than $0.0005 \mathrm{mgrm}$; stronger than $0.0003 \mathrm{mgrm}$. and not much different from $0.0004 \mathrm{mgrm}$., so it was assayed at $0.0008 \mathrm{mgrm}$. in 1 c.c. in diluted solution, i.e. $0.0016 \mathrm{mgrm}$. in 1 c.c. of original extract.

E.L. $\times 2$ : Stronger than $0.0002 \mathrm{mgrm}$. and $0.00025 \mathrm{mgrm}$. (Obs. $12 \& 13$ ) and almost as strong as $0.0003 \mathrm{mgrm}$. It was therefore assayed at $0.0006 \mathrm{mgrm}$. in 1 c.c. of diluted solution, i.e. $0.0012 \mathrm{mgrm}$. in 1 c.c. of original extract. 
Epinephrine concentration and output determined by rabbit intestine segment:

\begin{tabular}{|c|c|c|c|c|c|c|c|}
\hline \multirow{3}{*}{$\begin{array}{l}\text { No. of cava } \\
\text { pocket } \\
\text { specimen }\end{array}$} & \multicolumn{4}{|c|}{ Blood flow (c.c.) } & \multicolumn{3}{|c|}{$\begin{array}{c}\text { Epinephrine output } \\
\text { (mgrm.) }\end{array}$} \\
\hline & \multirow{2}{*}{ Quantity } & \multirow{2}{*}{$\begin{array}{c}\text { Duration } \\
\text { of } \\
\text { collection } \\
\text { (sec) }\end{array}$} & \multicolumn{2}{|c|}{$\begin{array}{l}\text { Quantity } \\
\text { per min. }\end{array}$} & \multirow{2}{*}{$\begin{array}{l}\text { Quantity } \\
\text { contained } \\
\text { in } 1 \text { c.c. }\end{array}$} & \multicolumn{2}{|c|}{$\begin{array}{l}\text { Output } \\
\text { per min. }\end{array}$} \\
\hline & & & $\underset{\text { animal }}{\text { per }}$ & $\begin{array}{l}\text { per } \\
\text { kilo }\end{array}$ & & $\begin{array}{c}\text { per } \\
\text { animal }\end{array}$ & $\begin{array}{l}\text { per } \\
\text { kilo }\end{array}$ \\
\hline I & 3.5 & 180 & 1.16 & 0.38 & 0.0013 & 0.0015 & 0.00043 \\
\hline II & 2.6 & 150 & 1.04 & 0.33 & 0.0011 & 0.0011 & 0.00031 \\
\hline
\end{tabular}

Epinephrine content per grm. tissue of the gland determined by rabbit intestine segment :

\begin{tabular}{c|c}
\hline R. or L. extract & $\begin{array}{c}\text { Epinephrine content per grm. tissue } \\
\text { of the gland (mgrm.) }\end{array}$ \\
\hline E.R. & 0.16 \\
E.L. & 0.09
\end{tabular}

Ratio of the two assays of the cava pocket blood:

\begin{tabular}{|c|c|c|c|}
\hline \multirow{2}{*}{$\begin{array}{c}\text { No. of cava } \\
\text { pocket specimen }\end{array}$} & \multicolumn{2}{|c|}{ Epinephrine concentration (mgrm. in 1 c.c.) } & \multirow{2}{*}{$\begin{array}{c}\text { Ratio } \\
\text { (Pupil : Intestine) }\end{array}$} \\
\hline & Pupil & Intestine & \\
\hline $\begin{array}{r}\text { I } \\
\text { II }\end{array}$ & $\begin{array}{l}0.0011 \\
0.0009\end{array}$ & $\begin{array}{l}0.0013 \\
0.0011\end{array}$ & $\begin{array}{l}1: 1.2 \\
1: 1.2\end{array}$ \\
\hline
\end{tabular}

Epinephrine content per grm. tissue of the gland determined by colorimetoric procedure of Folin, Cannon and Denis :

\begin{tabular}{c|c}
\hline R. or L. extract & $\begin{array}{c}\text { Epinephrine content per grm. tissue } \\
\text { of the gland (mgrm.) }\end{array}$ \\
\hline E.R. & 0.41 \\
E.L. & 0.59
\end{tabular}

Epinephrine content per grm. tissue of the gland determined by the three assays:

\begin{tabular}{|c|c|c|c|c|c|c|}
\hline \multirow{2}{*}{$\begin{array}{l}\text { R. or } \mathrm{L} . \\
\text { extract }\end{array}$} & \multicolumn{3}{|c|}{$\begin{array}{l}\text { Epinephrine content (in mgrms. } \\
\text { per grm. tissue) deternined by }\end{array}$} & \multicolumn{3}{|c|}{ Ratio of values determined by } \\
\hline & Folin & Eye & Intestine & $\begin{array}{c}\text { Eye : } \\
\text { Intestine }\end{array}$ & $\begin{array}{l}\text { Eye: } \\
\text { Folin }\end{array}$ & $\begin{array}{l}\text { Folin : } \\
\text { Intestine }\end{array}$ \\
\hline $\begin{array}{l}\text { E.R. } \\
\text { E.L. }\end{array}$ & $\begin{array}{l}0.41 \\
0.59\end{array}$ & $\begin{array}{l}0.18 \\
0.21\end{array}$ & $\begin{array}{l}0.16 \\
0.09\end{array}$ & $\begin{array}{l}1.1: 1 \\
1.5: 1\end{array}$ & $\begin{array}{l}1: 2.3 \\
1: 2.8\end{array}$ & $\begin{array}{l}2.5: 1 \\
4.1: 1\end{array}$ \\
\hline
\end{tabular}




\section{TAble I.}

Comparison of the epinephrine content in the suprarenal rein blood from pregnant rabbits by means of the rabbit intestine. segment and the cat paradoxical eye reaction.

\begin{tabular}{|c|c|c|c|c|c|c|c|c|c|}
\hline \multirow{3}{*}{ No. of animal } & \multirow{3}{*}{ 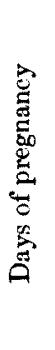 } & \multirow{3}{*}{ 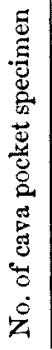 } & \multirow{2}{*}{\multicolumn{2}{|c|}{$\begin{array}{c}\begin{array}{c}\text { Blood flow } \\
\text { (c.c.) }\end{array} \\
\begin{array}{c}\text { Quantity } \\
\text { per } \\
\text { minute }\end{array}\end{array}$}} & \multirow{2}{*}{\multicolumn{2}{|c|}{$\begin{array}{c}\begin{array}{c}\text { Assay by } \\
\text { paradoxical eye } \\
\text { reaction of cat }\end{array} \\
\begin{array}{c}\text { Epinephrine } \\
\text { output } \\
\text { (mgrm.) }\end{array}\end{array}$}} & \multirow{2}{*}{\multicolumn{2}{|c|}{$\begin{array}{c}\text { Assay by } \\
\text { intestine strip } \\
\text { of rabbit } \\
\text { Epinepbrine } \\
\text { output } \\
\text { (mgrm.) }\end{array}$}} & \multirow{3}{*}{ 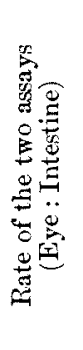 } \\
\hline & & & & & & & & & \\
\hline & & & 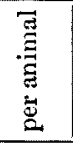 & $\frac{0}{3}$ & 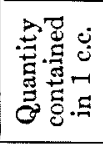 & 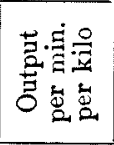 & 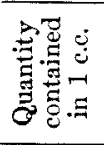 & 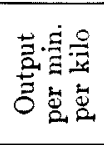 & \\
\hline $\begin{array}{c}\text { 35. } 2.23 \text { kilos } \\
\text { 4. II. } 1929\end{array}$ & 4 & $\mathrm{I}$ & 0.4 & 0.18 & 0.0012 & 0.00022 & 0.0006 & 0.00011 & $2.0: 1$ \\
\hline $\begin{array}{l}\text { 36. } 2.57 \text { kilos } \\
\text { 7. II. } 1929\end{array}$ & 5 & $\begin{array}{c}\text { I } \\
\text { II }\end{array}$ & $\begin{array}{l}1.0 \\
0.7\end{array}$ & $\begin{array}{l}0.39 \\
0.27\end{array}$ & $\begin{array}{l}0.0012 \\
0.0013\end{array}$ & $\begin{array}{l}0.00047 \\
0.00035\end{array}$ & $\begin{array}{l}0.0007 \\
0.00075\end{array}$ & $\begin{array}{l}0.00027 \\
0.0002\end{array}$ & $\begin{array}{l}1.7: 1 \\
1.7: 1\end{array}$ \\
\hline $\begin{array}{l}\text { 42. } 2.83 \text { kilos } \\
\text { 7. III. } 1929\end{array}$ & 6 & I & 2.6 & 0.92 & $0.000 \mathrm{~s}$ & 0.00074 & 0.00035 & 0.00032 & $2.3: 1$ \\
\hline $\begin{array}{l}\text { 37. } 2.46 \text { kilos } \\
\text { 14. II. } 1929\end{array}$ & 8 & I & 1.15 & 0.47 & 0.0014 & 0.00065 & 0.0009 & 0.00042 & $1.5: 1$ \\
\hline $\begin{array}{l}\text { 43. } 2.45 \text { kilos } \\
\text { 14. III. } 1929\end{array}$ & 9 & I & $\begin{array}{l}2.15 \\
1.5\end{array}$ & $\begin{array}{l}0.88 \\
0.6\end{array}$ & $\begin{array}{l}0.0006 \\
0.0007\end{array}$ & $\begin{array}{l}0.00053 \\
0.00043\end{array}$ & $\begin{array}{l}0.0004 \\
0.00055\end{array}$ & $\begin{array}{l}0.00035 \\
0.00032\end{array}$ & $\begin{array}{l}1.5: 1 \\
1.3: 1\end{array}$ \\
\hline $\begin{array}{l}14.2 \\
25 . \mathrm{V}\end{array}$ & 10 & $\underset{I I}{I}$ & $\begin{array}{l}1.0 \\
1.0\end{array}$ & & $\begin{array}{l}0.0008 \\
0.0008\end{array}$ & $\begin{array}{l}0.00034 \\
0.00034\end{array}$ & $\begin{array}{l}0.0005 \\
0.0005\end{array}$ & $\begin{array}{l}0.00021 \\
0.00021\end{array}$ & $\begin{array}{l}1.6: 1 \\
1.6: 1\end{array}$ \\
\hline $\begin{array}{l}38.2 .6 \text { kilos } \\
\text { 14. II. } 1929\end{array}$ & 12 & I & 1.32 & 0.51 & 0.0010 & 051 & 0.0008 & 0.00042 & $1.2: 1$ \\
\hline $\begin{array}{l}\text { 34. } 2.38 \text { kilos } \\
\text { 4. II. } 1929\end{array}$ & 13 & I & 0.78 & 0.83 & 0.0012 & 0.00039 & 0.0010 & 0.00033 & $1.2: 1$ \\
\hline $\begin{array}{l}\text { 41. } 2.17 \text { kilos } \\
\text { 7. III. } 1929\end{array}$ & 14 & I & 1.1 & 0.5 & 0.0016 & 0.00083 & 0.0008 & 0.00041 & $2.0: 1$ \\
\hline $\begin{array}{l}4 \text { kilos } \\
.1928\end{array}$ & 15 & $\begin{array}{c}\text { I } \\
\text { II }\end{array}$ & $\begin{array}{l}1.5 \\
1.5\end{array}$ & $\begin{array}{l}0.64 \\
0.64\end{array}$ & $\begin{array}{l}0.0006 \\
0.0006\end{array}$ & $\begin{array}{l}0.00038 \\
0.00038\end{array}$ & $\begin{array}{l}0.00045 \\
0.00045\end{array}$ & $\begin{array}{l}0.00029 \\
0.00029\end{array}$ & $\begin{array}{l}1.3: 1 \\
1.3: 1\end{array}$ \\
\hline $\begin{array}{l}\text { 39. } 2.66 \text { kilos } \\
\text { 2. III. } 1929\end{array}$ & 16 & I & 1.15 & 0.43 & 0.0008 & 0.00035 & 0.0006 & 0.00026 & $1.3: 1$ \\
\hline $\begin{array}{l}\text { 25. } 2.61 \text { kilos } \\
\text { 12. XI. } 1928\end{array}$ & 17 & I & $\begin{array}{l}0.9 \\
0.87\end{array}$ & $\begin{array}{l}0.34 \\
0.33\end{array}$ & $\begin{array}{l}0.0006 \\
0.0006\end{array}$ & $\begin{array}{l}0.00021 \\
0.0002\end{array}$ & $\begin{array}{l}0.00045 \\
0.00045\end{array}$ & $\begin{array}{l}0.00015 \\
0.00015\end{array}$ & $\begin{array}{l}1.3: 1 \\
1.3: 1\end{array}$ \\
\hline $\begin{array}{l}\text { 24. } 2.92 \text { kilos } \\
\text { 8. XI. } 1928\end{array}$ & 20 & I & 0.94 & 0.31 & 0.0006 & 0.00019 & 0.00045 & 0.00014 & $1.3: 1$ \\
\hline $\begin{array}{l}\text { 23. } 2.74 \text { kilos } \\
\text { 8. XI. } 1928\end{array}$ & 20 & I & $\begin{array}{l}2.0 \\
2.0\end{array}$ & $\begin{array}{l}0.73 \\
0.73\end{array}$ & $\begin{array}{l}0.0008 \\
0.0008\end{array}$ & $\begin{array}{l}0.00058 \\
0.00058\end{array}$ & $\begin{array}{l}0.00045 \\
0.00045\end{array}$ & $\begin{array}{l}0.00033 \\
0.00033\end{array}$ & $\begin{array}{l}1.8: 1 \\
1.8: 1\end{array}$ \\
\hline
\end{tabular}




\begin{tabular}{|c|c|c|c|c|c|c|c|c|c|}
\hline \multirow{3}{*}{ No. of animal } & \multirow{3}{*}{ 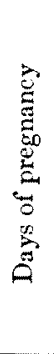 } & \multirow{3}{*}{ 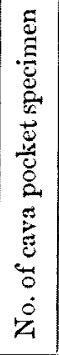 } & \multirow{2}{*}{\multicolumn{2}{|c|}{$\begin{array}{c}\begin{array}{c}\text { Blood flow } \\
\text { (c.c.) }\end{array} \\
\begin{array}{c}\text { Quantity } \\
\text { per } \\
\text { minute }\end{array}\end{array}$}} & \multirow{2}{*}{\multicolumn{2}{|c|}{$\begin{array}{c}\text { Assay by } \\
\text { paradoxical eye } \\
\text { reaction of cat } \\
\begin{array}{c}\text { Epinephrine } \\
\text { output } \\
\text { (mgrm.) }\end{array}\end{array}$}} & \multirow{2}{*}{\multicolumn{2}{|c|}{$\begin{array}{c}\begin{array}{c}\text { Assay by } \\
\text { intestine strip } \\
\text { of rabbit }\end{array} \\
\begin{array}{c}\text { Epinephrine } \\
\text { output } \\
\text { (mgrm.) }\end{array}\end{array}$}} & \multirow{3}{*}{ 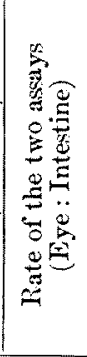 } \\
\hline & & & & & & & & & \\
\hline & & & 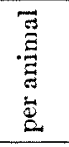 & $\stackrel{ }{\stackrel{3}{3}}$ & 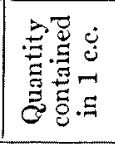 & 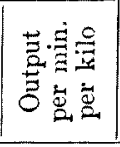 & 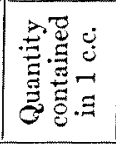 & 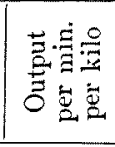 & \\
\hline $\begin{array}{l}\text { 31. } 2.25 \text { kilos } \\
\text { 27. XII. } 1928\end{array}$ & 20 & I & 0.9 & 0.4 & 0.0012 & 0.00049 & 0.0010 & 0.0004 & $1.2: 1$ \\
\hline $\begin{array}{l}\text { 22. } 2.75 \text { kilos } \\
\text { 5. XI. } 1928\end{array}$ & 21 & $\mathrm{I}$ & 1.5 & 0.55 & 0.0006 & 0.00033 & 0.0006 & 0.00083 & $1: 1$ \\
\hline $\begin{array}{l}\text { 26. } 2.86 \text { kilos } \\
\text { 19. XI. } 1928\end{array}$ & 21 & I & 0.73 & 0.25 & 0.0019 & 0.00049 & 0.0018 & 0.00045 & $1^{+}: 1$ \\
\hline $\begin{array}{l}\text { 25. } 1.66 \text { kilos } \\
\text { 4. XII. } 1928\end{array}$ & 22 & I & 1.0 & 0.6 & 0.0004 & 0.00024 & 0.0005 & 0.0003 & $1: 1.2$ \\
\hline $\begin{array}{l}\text { 29. } 2.7 \text { kilos } \\
\text { 11. XII. } 1928\end{array}$ & 28 & $\mathrm{I}$ & 0.55 & 0.2 & 0.0018 & 0.00037 & 0.0020 & 0.00041 & $1: 1.1$ \\
\hline $\begin{array}{l}\text { 44. } 2.6 \text { kilos } \\
\text { 14. III. } 1929\end{array}$ & 22 & I & 1.25 & 0.48 & 0.0008 & 0.00038 & 0.00095 & 0.00046 & $1: 1.2$ \\
\hline $\begin{array}{l}\text { 47. } 2.61 \text { kilos } \\
\text { 11. IV. } 1929\end{array}$ & 22 & $\mathrm{I}$ & 1.0 & 0.88 & 0.0011 & 0.00042 & 0.0012 & 0,00046 & $1: 1.1$ \\
\hline $\begin{array}{l}\text { 7. } 2.1 \text { kilos } \\
\text { 31. V. } 1928\end{array}$ & 23 & I & 1.25 & 0.59 & $0.000 \mathrm{~s}$ & 0.00048 & 0.0011 & 0.00067 & $1: 1.4$ \\
\hline $\begin{array}{l}\text { 10. } 2.17 \text { kilos } \\
\text { 19. VII. } 1928\end{array}$ & 23 & I & 1.1 & 0.5 & 0.0006 & 0.0008 & 0.0002 & 0.0001 & $3.0: 1$ \\
\hline $\begin{array}{l}40.2 .29 \mathrm{kilos} \\
\text { 2. III. } 1929\end{array}$ & $2: 3$ & $I$ & 1.15 & 0.5 & 0.0009 & 0.00044 & 0.0012 & 0.00053 & $1: 1.3$ \\
\hline $\begin{array}{l}\text { 2. } 2.35 \mathrm{kilos} \\
\text { 15. X. } 1927\end{array}$ & 24 & $\stackrel{\text { I }}{\text { II }}$ & $\begin{array}{l}1.0 \\
1.0\end{array}$ & $\begin{array}{l}0.42 \\
0.42\end{array}$ & $\begin{array}{l}0.000 \mathrm{~s} \\
0.0008\end{array}$ & $\begin{array}{l}0.00034 \\
0.00034\end{array}$ & $\begin{array}{l}0.0011 \\
0.0011\end{array}$ & $\begin{array}{l}0.00047 \\
0.00047\end{array}$ & $\begin{array}{l}1: 1.4 \\
1: 1.4\end{array}$ \\
\hline $\begin{array}{l}\text { 9. } 3.51 \text { kilos } \\
\text { 11. VJI. } 1928\end{array}$ & 24 & $\begin{array}{c}\text { I } \\
\text { II }\end{array}$ & $\begin{array}{l}1.16 \\
1.04\end{array}$ & $\begin{array}{l}0.38 \\
0.3\end{array}$ & $\begin{array}{l}0.0011 \\
0.0009\end{array}$ & $\begin{array}{l}0.00037 \\
0.00027\end{array}$ & $\begin{array}{l}0.0018 \\
0.0011\end{array}$ & $\begin{array}{l}0.00043 \\
0.00031\end{array}$ & $\begin{array}{l}1: 1.2 \\
1: 1.2\end{array}$ \\
\hline $\begin{array}{c}\text { 20. } 2.44 \text { kilos } \\
\text { 8. X. } 1928\end{array}$ & 24 & I & 0.46 & 0.19 & 0.0010 & 0.00019 & 0.0014 & 0.00026 & $1: 1.4$ \\
\hline $\begin{array}{l}\text { 49. 2.0 kilos } \\
\text { 30. IV. } 1929\end{array}$ & 24 & I & 0.57 & 0.28 & 0.0008 & 0.000085 & 0.00035 & 0.0001 & $1: 1.2$ \\
\hline $\begin{array}{l}\text { 12. } 2.33 \text { kilos } \\
30 . \text { VII. } 1928\end{array}$ & 25 & I & 1.7 & 0.73 & 0.0002 & 0.00014 & 0.0004 & 0.00029 & $1: 2.0$ \\
\hline $\begin{array}{l}\text { 13. } 2.1 \mathrm{kilos} \\
30 . \text { VII. } 1928\end{array}$ & 25 & I & 0.9 & 0.43 & 0.0012 & 0.00055 & 0.0015 & 0.00062 & $1: 1.2$ \\
\hline
\end{tabular}




\begin{tabular}{|c|c|c|c|c|c|c|c|c|c|}
\hline \multirow{3}{*}{ No. of animal } & \multirow{3}{*}{ 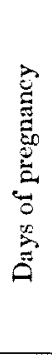 } & \multirow{3}{*}{ 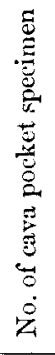 } & \multirow{2}{*}{\multicolumn{2}{|c|}{$\begin{array}{c}\begin{array}{c}\text { Blood flow } \\
\text { (c.c.) }\end{array} \\
\begin{array}{c}\text { Quantity } \\
\text { per } \\
\text { minute }\end{array}\end{array}$}} & \multirow{2}{*}{\multicolumn{2}{|c|}{$\begin{array}{c}\begin{array}{c}\text { Assay by } \\
\text { paradoxical eye } \\
\text { reaction of cat }\end{array} \\
\begin{array}{c}\text { Epinephrine } \\
\text { output } \\
\text { (mgrm.) }\end{array}\end{array}$}} & \multirow{2}{*}{\multicolumn{2}{|c|}{$\begin{array}{c}\text { Assay by } \\
\text { intestine strip } \\
\text { of rabbit } \\
\text { Epinephrine } \\
\text { output } \\
\text { (mgrm.) }\end{array}$}} & \multirow{3}{*}{ 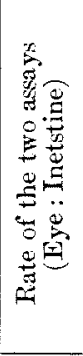 } \\
\hline & & & & & & & & & \\
\hline & & & 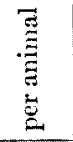 & $\frac{\stackrel{0}{E}}{: 3}$ & 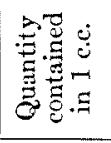 & 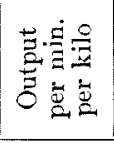 & 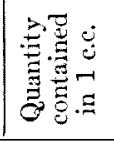 & 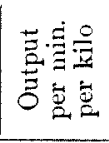 & \\
\hline $\begin{array}{l}\text { 18. } 2.39 \text { kilos } \\
\text { 29. IX. } 1928\end{array}$ & 25 & I & 1.5 & 0.62 & 0.0004 & 0.00025 & 0.0008 & 0.00019 & $1.8: 1$ \\
\hline $\begin{array}{l}\text { 45. } 2.59 \text { kilos } \\
28 . \text { III. } 1929\end{array}$ & 25 & $\begin{array}{l}\text { I } \\
\text { II }\end{array}$ & $\begin{array}{l}1.5 \\
1.3\end{array}$ & $\begin{array}{l}0.58 \\
0.5\end{array}$ & $\begin{array}{l}0.0005 \\
0.0005\end{array}$ & $\begin{array}{l}0.00029 \\
0.00025\end{array}$ & $\begin{array}{l}0.0006 \\
0.0006\end{array}$ & $\begin{array}{l}0.00035 \\
0.0003\end{array}$ & $\begin{array}{l}1: 1.2 \\
1: 1.2\end{array}$ \\
\hline & 26 & $\stackrel{\text { I }}{I I}$ & $\begin{array}{l}1.0 \\
0.75\end{array}$ & & $\begin{array}{l}0.0008 \\
0.0012\end{array}$ & $\begin{array}{l}0.00042 \\
0.0 j 047\end{array}$ & $\begin{array}{l}0.0011 \\
0.0016\end{array}$ & & $\begin{array}{l}1: 1.4 \\
1: 1.3\end{array}$ \\
\hline & $\$ 6$ & $\underset{\mathrm{II}}{\mathrm{I}}$ & $\begin{array}{l}0.95 \\
1.0\end{array}$ & & $\begin{array}{l}0.0008 \\
0.0008\end{array}$ & $\begin{array}{l}0.00031 \\
0.00039\end{array}$ & $\begin{array}{l}0.0018 \\
0.0016\end{array}$ & & $\begin{array}{l}1: 2.2 \\
1: 2.0\end{array}$ \\
\hline $\begin{array}{l}\text { 4. } 2.45 \text { kilos } \\
28.11 .192 \mathrm{~s}\end{array}$ & 26 & I & 1.0 & 0.41 & 0.0012 & 0.00049 & 0.0014 & 0057 & $1: 1.2$ \\
\hline $\begin{array}{l}\text { S. } 2.12 \text { kilos } \\
\text { s1. V. } 1929\end{array}$ & 26 & I & 1.2 & 0.56 & 0.0002 & 0.00011 & 0.0005 & 0.00028 & $1: 2.2$ \\
\hline $\begin{array}{l}\text { 11. } 2.3 \mathrm{kilos} \\
\text { 19. VII. } 192 \mathrm{~s}\end{array}$ & 27 & I & 1.1 & 0.48 & 0.0012 & 0.00057 & 0.004 & 0.00065 & $1: 1.2$ \\
\hline $\begin{array}{l}\text { 15. } 2.36 \text { kilos } \\
\text { 3. IX. } 1928\end{array}$ & 27 & $\underset{I I}{I}$ & $\begin{array}{l}2.2 \\
2.4\end{array}$ & $\begin{array}{l}0.93 \\
1.0\end{array}$ & $\begin{array}{l}0.000 \mathrm{~S} \\
0.0008\end{array}$ & $\begin{array}{l}0.00076 \\
0.0008\end{array}$ & $\begin{array}{l}0.0013 \\
0.0013\end{array}$ & & $\begin{array}{l}1: 1.5 \\
1: 1.5\end{array}$ \\
\hline $\begin{array}{l}\text { 16. 2.55 kilos } \\
\text { 10. IX. } 1928\end{array}$ & 27 & $I$ & 0.65 & 0.25 & 0.0004 & 0.0001 & 0.0005 & 0.00013 & $1: 1.2$ \\
\hline $\begin{array}{l}21.8 .06 \text { kilos } \\
22 . \mathrm{X} .1928\end{array}$ & 27 & $\underset{I I}{I}$ & $\begin{array}{l}1.0 \\
1.1\end{array}$ & $\begin{array}{l}0.33 \\
0.36\end{array}$ & $\begin{array}{l}0.0010 \\
0.0008\end{array}$ & $\begin{array}{l}0.00022 \\
0.00029\end{array}$ & $\begin{array}{l}0.0017 \\
0.0013\end{array}$ & $\begin{array}{l}0.00056 \\
0.00046\end{array}$ & $\begin{array}{l}1: 1.7 \\
1: 1.7\end{array}$ \\
\hline $\begin{array}{l}17.3 .1 \text { kilos } \\
24.1 X .1928\end{array}$ & 28 & $\underset{I I}{\text { II }}$ & $\begin{array}{l}1.6 \\
2.0\end{array}$ & $\begin{array}{l}0.52 \\
0.65\end{array}$ & $\begin{array}{l}0.0004 \\
0.0003\end{array}$ & $\begin{array}{l}0.00021 \\
0.00019\end{array}$ & $\begin{array}{l}0.0006 \\
0.0004\end{array}$ & & $\begin{array}{l}1: 1.5 \\
1: 1.5\end{array}$ \\
\hline $\begin{array}{l}\text { 50. } 1.97 \mathrm{kilos} \\
\text { 9. V. } 19 \pm 9\end{array}$ & 28 & I & 0.63 & 0.32 & $0.000 \mathrm{~s}$ & 0.00025 & 0.0009 & 0.00029 & $1: 1.1$ \\
\hline $\begin{array}{l}\text { 5. } 2.69 \text { kilos } \\
24 \text { IV. } 1928\end{array}$ & 29 & I & 1.0 & 0.37 & 0.0004 & 0.00015 & 0.0004 & 0.00015 & $1: 1$ \\
\hline $\begin{array}{l}\text { 30. } 2.47 \text { kilos } \\
\text { 18. XII. } 1928\end{array}$ & 29 & I & 1.15 & 0.46 & 0.0009 & 0.0004 & 0.0009 & 0.0004 & $1: 1$ \\
\hline $\begin{array}{l}\text { 19. } 2.36 \text { kilos } \\
\text { 29. IX. } 1928\end{array}$ & 30 & I & $\begin{array}{l}0.86 \\
0.51\end{array}$ & $\begin{array}{l}0.36 \\
0.21\end{array}$ & $\begin{array}{l}0.0008 \\
0.0008\end{array}$ & $\begin{array}{l}0.00029 \\
0.00017\end{array}$ & $\begin{array}{l}0.0006 \\
0.0007\end{array}$ & $\begin{array}{l}0.00022 \\
0.00015\end{array}$ & $\begin{array}{l}1.3: 1 \\
1.1: 1\end{array}$ \\
\hline $\begin{array}{l}\text { 27. } 2.6 \text { kilos } \\
29 . \text { XI. } 1928\end{array}$ & 30 & I & 0.9 & 0.35 & 0.0015 & 0.00054 & 0.0010 & 0.00035 & $1.5: 1$ \\
\hline
\end{tabular}




\begin{tabular}{|c|c|c|c|c|c|c|c|c|c|}
\hline \multirow{3}{*}{ No. of animal } & \multirow{3}{*}{ 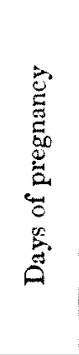 } & \multirow{3}{*}{ 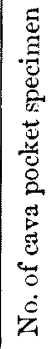 } & \multirow{2}{*}{\multicolumn{2}{|c|}{$\begin{array}{c}\begin{array}{c}\text { Blood flow } \\
\text { (c.e.) }\end{array} \\
\begin{array}{c}\text { Quantity } \\
\text { per } \\
\text { minute }\end{array}\end{array}$}} & \multirow{2}{*}{\multicolumn{2}{|c|}{$\begin{array}{c}\begin{array}{c}\text { Assay by } \\
\text { paradoxical eye } \\
\text { reaction of cat }\end{array} \\
\begin{array}{c}\text { Epinephrine } \\
\text { output } \\
\text { (mgrm.) }\end{array}\end{array}$}} & \multirow{2}{*}{\multicolumn{2}{|c|}{$\begin{array}{c}\begin{array}{c}\text { Assay by } \\
\text { intestine strip } \\
\text { of rabbit }\end{array} \\
\begin{array}{c}\text { Epinephrine } \\
\text { output } \\
\text { (mgrn.) }\end{array}\end{array}$}} & \multirow{3}{*}{ 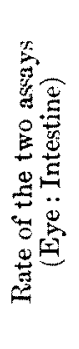 } \\
\hline & & & & & & & & & \\
\hline & & & 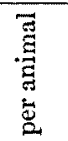 & $\frac{\stackrel{0}{ت}}{\ddot{3}}$ & 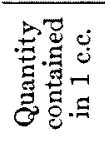 & 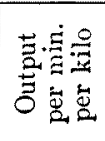 & 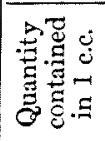 & 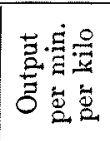 & \\
\hline $\begin{array}{l}\text { 33. } 2.69 \text { kilos } \\
\text { 19. I. } 1929\end{array}$ & $\begin{array}{c}\text { ca. } 8 \\
\text { hours } \\
\text { after }\end{array}$ & I & 0.95 & 0.36 & 0.0024 & 0.00085 & 0.0008 & 0.00028 & $3.0: 1$ \\
\hline $\begin{array}{l}\text { 32. } 2.55 \text { kilos } \\
\text { 14. I. } 1929\end{array}$ & $\begin{array}{l}2 \text { days } \\
\text { after }\end{array}$ & $\underset{\text { II }}{\text { I }}$ & $\begin{array}{l}1.0 \\
1.0\end{array}$ & $\begin{array}{l}0.39 \\
0.39\end{array}$ & 0.0006 & $\begin{array}{l}0.00024 \\
0.00024\end{array}$ & $\begin{array}{l}0.0002 \\
0.0002\end{array}$ & $\begin{array}{l}0.00079 \\
0.00079\end{array}$ & $\begin{array}{l}3.0: 1 \\
3.0: 1\end{array}$ \\
\hline
\end{tabular}

$1: 1.3$ (from 18 pairs of the estimations), rabbits $10 \& 18$ being excluded from the averaging. In Rabbit 10 the ratio was $3.0: 1$, this was due to an extraordinarily small value obtained by the rabbit intestine strip method. The concentration noted by that method was $0.0002 \mathrm{mgrm}$. in 1 c.c. blood, - the lowest value obtained from the pregnant rabbits; such low value was also found only seldom with the normal rabbits. We have at present no explanation to account for this discrepancy of the two cases from the remainder, only we feel it to be justifiable to deal with these two cases as the exception because of the small frequency of occurrence. Summarizing the ratios for the 26 th to 28 th day of the pregnancy, the average was computed as $1: 1.5$.

In two cases of the 29 th day of the pregnancy the values obtained by both methods again closely agreed with each other, and on the 30th day the ratio (Eye: Intestine) approached that of the non-pregnant stage, that is $1.3: 1(1.1: 1$ to $1.5: 1)$, or the value yielded by the rabbit intestine became comparatively less than that by the denervated eye. In the rabbits, which were experimented on 8 hours and 2 days after delivery, the value by the intestine was found far less than that by the other, the ratio being calculated as $3.0: 1$ for both rabbits ( 3 pairs of determinations).

Now turning to the epinephrine output rate, which was calculated from the epinephrine content determined by means of the rabbit intestine, it is computed as follows for the several periods of the pregnancy :-For the 4th to the 10 th days the mean rate $0.00027 \mathrm{mgrm}$. per kilo per minute and that from the 12 th to the 20 th day $0.0003 \mathrm{mgrm}$. per kilo per minute. Both these values do not much differ from the mean rate estimated for the non-preg- 
nant rabbit in the previous paper which was reckoned as $0.0003 \mathrm{mgrm}$. per kilo per minute. The rate from the 21 st to the 25 th day of pregnancy was $0.0004 \mathrm{mgrm}$. (Rabbits 10 and 18 were excluded from the averaging because of the abnormal ratio of the epinephrine determined by the two methods) and that from the 26th to the 28 th day 0.00053 mgrm. per kilo per minute. Both values, especially the latter decidedly exceeded the rate for the non-pregnant animal. But on the 29th and the 30th day of the pregnancy and on the 1st puerperal day it was inclined to reduce, that is about $0.00027 \mathrm{mgrm}$. per kilo per minute was the mean ratio for each of these days, and on the 2nd puerperal day it was about $0.00008 \mathrm{mgrm}$. per kilo per minute.

Taking the data altogether it is sure that the epinephrine (Intestine) output rate of rabbits was definitely increased on from the 26th to the 28th day of the pregnancy.

If now the values of the epinephrine determined by the denervated pupil of the cat be taken into account the reverse becomes true. The velocity of the epinephrine (Eye) liberation was rather reduced in comparison with that in the non-pregnant stage which was determined previously as from 0.00023 to 0.0014 mgrm., mean $0.00066 \mathrm{mgrm}$. per kilo per minute. The velocity from the th th the 10 th day of the pregnancy was $0.00047 \mathrm{mgrm}$. per kilo per minute, from the 12 th to the 20 th day 0.00043 mgrm., from the 21st to the 25th day (Nos. $10 \& 18$ being excluded) $0.00034 \mathrm{mgrm}$. and from the 26 th to the 28 th $0.00036 \mathrm{mgrm}$., on the 29th day 0.00027 mgrm., on the 30 th day 0.00038 mgrm. 8 hours after delivery 0.00085 mgrm., and 2 days thereafter $0.00024 \mathrm{mgrm}$. The values of the latter four periods are based on a very small number of experiments.

Only from the outcome yielded by means of the rabbit strip ruethod are we able to state an acceleration of the epinephrine output during pregnancy, but the paradoxical pupil reaction tells something others. Since we have several pieces of evidence that the rabbit intestine strip method, the cat paradoxical pupil method, etc., which are nowadays taken as applicable for determining the epinephrine existing in the blood, yielded different values of epinephrine for the bloods of various kinds of animal, when they run in parallel in determining the epinephrine in one and the same blood sample with pure adrenaline chloride solution as the standard, we are now in this laboratory practising the pupil reaction simultaneously with the rabbit intestine strip method, and have found it to corroborate the latter metbod in the case of dogs and cats, as the recent papers show; but with rabbits the matter is quite different as the present paper indicates.

The reports ${ }^{2}$ that dispersion of epinephrine or an allied substance into the general blood stream during pregnancy is accelerated have not been publicated by subsquent workers, the

2) If. Neu, Med. Klinik, 1910, 1813; If. m. W., 1911, 1810. 
same procedures (the rabbit uterus and the frog leg ressels) being applied. ${ }^{3}$ ) It is however a well substantiated fact that the suprarenal gland undergoes some morphological changes during pregnancy.

In order to show at one glance the alteration of the ability of the suprarenal vein blood to inhibit the rabbit intestine strip (epinephrine-Intestine) and to dilating the denervated pupil of the cat (epinephrine-Eye), taking place during the pregnant period, a figure is given here. It will speak for itself; there is no need of further explanation.

Fig. 2.

The fuctuation of the epinephrine content of the suprarenal rein blood of rabbits

during the pregnancy and after the delivery, determinable by means of the cat-paradoxical pupil reaction and the rabbit intestine strip.

A. Average value of epinephrine content determined by the denervated eye of cat.

B. Arerage value of epinephrine content determined by the rabbit intestine segment.

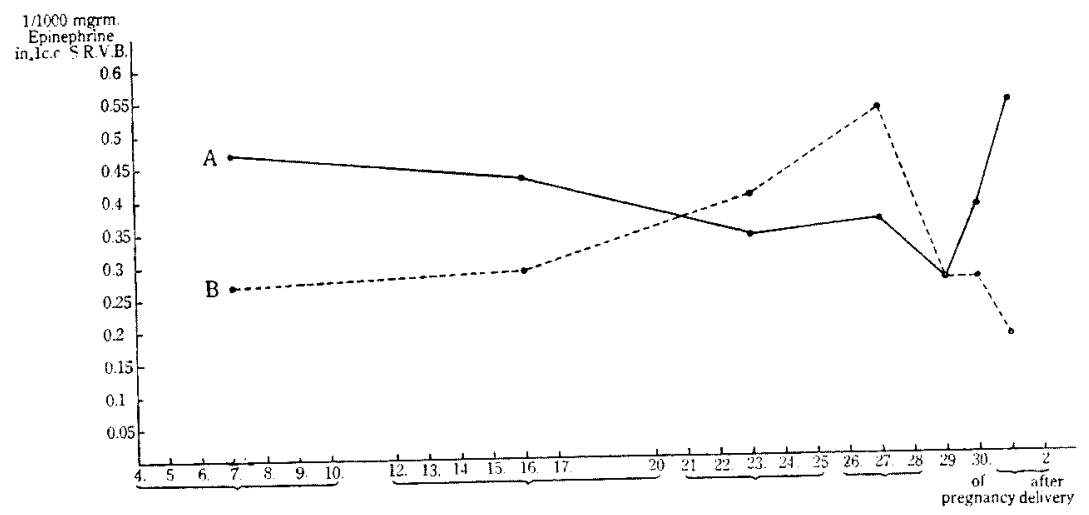

That the entero-inhibitory and the pupillo-dilatory ability do not develop in the same degree for one and the same sample of blood, not only of the rabbit, but also of the other species of animals, has been shown in several papers from this laboratory. In this respect however the suprarenal rein blood of the rabbit shows particular features differing from the others. These facts may be probably explained by assuming that the sympathomimetic power in the suprarenal vein blood and the gland extract is not due only to the adrenaline, but also to some other allied substances, each of which, for example, exerts sympathominetic influence upon the tissues in a different degree according to the tissues, and such substances are contained in various amounts in the suprarenal vein blood and in the suprarenal gland extract of various kinds of animal. Now the above given data occuring during the pregnancy of the rabbit may be explained by developing such supposition, that is by assuming alteration of the content of a certain substance or substances of such a nature during pregnancy. As a matter of course such a supposition is not deniable, viz. that the suprarenal

3) E. Bröking and P. Trendelenburg, Deut. Arch. f. kl. Med., 1911, 103, 181183; E. Neubauer and J. Norak, D. m. W., 1911, 2287. 
vein blood or the gland extract contains only adrenaline as the sympathomimetic agent, but there are some substances which exhibit an inhibitory influence upon the action of adrenaline in various ways in the long run. At present we know only that the adrenaline is prepared from the suprarenal gland or its medulla.

(2) Epinephrine in the Suprarenal Glands of the Pregnant Rabbits, Removed after Collection of Some

Cava Pocket Specimens.

At the termination of the experiments quoted in the foregoing chapter, the suprarenal glands were carefully removed, and extract was prepared from then according to Folin, Cannon and Denis. Epinephrine was estimated by means of both biological tests, and of the colorimetric procedure of Folin and others modified by Kodama. Only the colorimetric estimation was carried out the next morning. The results are summarized in Table II.

The values of epinephrine obtained by the three methods for one and the same sample did not coincide with each other. It was so previously with the gland extract of normal or non-pregnant rabbits, which were sacrificed only for estimating the epinephrine or treated just as in the present investigation': the ratios of the three values for one and the same extract were epinephrine-Eye : epinephrine-Intestine $1: 1-1: 1.7$, mean $1: 1.2$, epinephrine-Eye : epinephrine-Folin $1: 1.5-1: 9.2$, mean $1: 4.4$, and epinephrine-Folin : epinephrine-Intestine $1.4: 1-8.5: 1$, mean $3.7: 1$.

In the beginning of the pregnancy the ratio of epinephrine-Eye : epinephrine-Intestine did not differ from the case of control animals quoted in the previous paper, only the ratio in Rabbit 43 was quite dissimilar with the rest ; in this case epinephrine-Intestine was found remarkably small in the gland, thereby the reverse ratio resulted. In Rabbits 6 and 39, which were experimented on respectively on the 15 th and the 16th day of pregnancy, the ratio was also reverse, and in the latter, epinephrine-Intestine was contained in the gland in a small quantity. Such inverse ratio, that is a greater content of epinephrine-Eye over epinephrine-Intestine was found frequently with advancement of the pregnancy; it was observed in 2 cases out of 3 on the 22 nd day of pregnancy, in 1 case out of 3 on the $23 \mathrm{rd}$ day, in 2 out of 3 cases on the 24th day, in 3 out of 4 on the 25 th day, in 3 out of 4 of the 26 th day, in 2 out of 4 on the 27 th, in one of two on the 28 th, 29 th and 30 th days. There was inverse ratio in two cases examined 8 hours or 2 days after delivery respectively. 


\section{TABLE II.}

Comparison of the epinephrine content in the suprarenal extract from pregnant rabbit by means of rabbit intestine segment, cat paradoxical eye reaction and colorimetric method of Folin, Cannon and Denis.

\begin{tabular}{|c|c|c|c|c|c|c|c|c|}
\hline \multirow{2}{*}{$\begin{array}{c}\text { No. \& weight } \\
\text { of animal } \\
\& \text { date of } \\
\text { experiment }\end{array}$} & \multirow{2}{*}{ 莺泀 } & \multirow{2}{*}{ 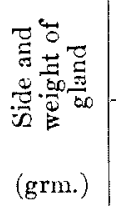 } & \multicolumn{3}{|c|}{$\begin{array}{c}\text { Epinephrine content } \\
\text { (in mgrms. per grm. tissue) } \\
\text { determined by }\end{array}$} & \multicolumn{3}{|c|}{$\begin{array}{l}\text { Ratios of values } \\
\text { determined by }\end{array}$} \\
\hline & & & Folin & Eye & $\begin{array}{l}\text { Intes- } \\
\text { tine }\end{array}$ & $\begin{array}{c}\text { Eye: } \\
\text { Intes- } \\
\text { tine }\end{array}$ & $\begin{array}{l}\text { Eye: } \\
\text { Folin }\end{array}$ & $\begin{array}{l}\text { Intes- } \\
\text { tine: } \\
\text { Folin }\end{array}$ \\
\hline $\begin{array}{l}\text { 35. } 2.23 \text { kilos } \\
\text { 4. II. } 1929\end{array}$ & 4 & $\begin{array}{ll}\text { R. } & 0.221 \\
\text { L. } & 0.214\end{array}$ & $\begin{array}{l}1.4 \\
1.8\end{array}$ & $\begin{array}{l}0.27 \\
0.26\end{array}$ & $\begin{array}{l}0.27 \\
0.26\end{array}$ & $\begin{array}{l}1: 1 \\
1: 1\end{array}$ & $\begin{array}{l}1: 5.2 \\
1: 5.6\end{array}$ & $\begin{array}{l}1: 5.2 \\
1: 5.6\end{array}$ \\
\hline $\begin{array}{l}\text { 36. } 2.57 \text { kilos } \\
\text { 7. II. } 1999\end{array}$ & 5 & L. 0.225 & 0.69 & 0.50 & 0.5 & $1: 1$ & $1: 1.5$ & $1: 1.5$ \\
\hline $\begin{array}{l}\text { 42. } 2.83 \text { kilos } \\
\text { 7. III. } 1929\end{array}$ & 6 & R. 0.256 & 0.71 & 0.29 & 0.54 & $1: 1.8$ & $1: 3.8$ & $1: 2.0$ \\
\hline $\begin{array}{l}\text { 37. } 2.46 \text { kilos } \\
\text { 14. II. } 1929\end{array}$ & 8 & 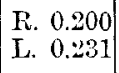 & $\begin{array}{l}1.39 \\
1.1\end{array}$ & $\begin{array}{l}0.30 \\
0.20\end{array}$ & $\begin{array}{l}0.37 \\
0.21\end{array}$ & $\begin{array}{l}1: 1.2 \\
1: 1^{+}\end{array}$ & $\begin{array}{l}1: 4.6 \\
1: 5.4\end{array}$ & $\begin{array}{l}1: 3.7 \\
1: 5.1\end{array}$ \\
\hline $\begin{array}{l}\text { 43. } 2.45 \text { kilos } \\
\text { 14. III. } 1929\end{array}$ & 9 & F. 0.435 & 0.72 & 0.21 & 0.09 & $2.4: 1$ & $1: 3.5$ & $1: 8.4$ \\
\hline $\begin{array}{c}\text { 14. } 2.35 \text { kilos } \\
25 . \text { VIII. } 1928\end{array}$ & 10 & $\begin{array}{ll}\text { R. } 0.17 \\
\text { L. } 0.15\end{array}$ & $\begin{array}{l}0.55 \\
0.9\end{array}$ & $\begin{array}{l}0.35 \\
0.40\end{array}$ & $\begin{array}{l}0.59 \\
0.4\end{array}$ & $\begin{array}{l}1: 1.7 \\
1: 1\end{array}$ & $\begin{array}{l}1: 2.4 \\
1: 2.2\end{array}$ & $\begin{array}{l}1: 1.4 \\
1: 2.2\end{array}$ \\
\hline $\begin{array}{l}38.2 .6 \text { kilos } \\
\text { 14. II. } 1929\end{array}$ & 12 & $\begin{array}{ll}\text { R. } & 0.287 \\
\text { L. } & 0.234\end{array}$ & $\begin{array}{l}0.78 \\
0.91\end{array}$ & $\begin{array}{l}0.12 \\
0.15\end{array}$ & $\begin{array}{l}0.12 \\
0.15\end{array}$ & $\begin{array}{l}1: 1 \\
1: 1.2\end{array}$ & $\begin{array}{l}1: 6.4 \\
1: 7.1\end{array}$ & $\begin{array}{l}1: 6.4 \\
1: 6.1\end{array}$ \\
\hline $\begin{array}{l}\text { 44. } 2.38 \text { kilos } \\
\text { 4. II. } 1929\end{array}$ & 13 & $\begin{array}{l}\text { R. } \\
\text { L. } 0.223 \\
0.234\end{array}$ & 1.2 & $\begin{array}{l}0.29 \\
0.21\end{array}$ & $\begin{array}{l}0.32 \\
0.21\end{array}$ & $\begin{array}{l}1: 1.1 \\
1: 1\end{array}$ & $\begin{array}{l}1: 4.7 \\
1: 5.5\end{array}$ & $\begin{array}{l}1: 4.1 \\
1: 5.5\end{array}$ \\
\hline $\begin{array}{l}\text { 41. } 2.17 \text { kilos } \\
\text { 7. III. } 1929\end{array}$ & 14 & L. 0.486 & 1.79 & 0.10 & 0.21 & $1: 2.0$ & $1: 16.6$ & $1: 8.3$ \\
\hline $\begin{array}{l}\text { 6. 2.34 kilos } \\
26 . \text { IV. } 1928\end{array}$ & 15 & $\begin{array}{ll}\text { R. } & 0.16 \\
\text { L. } & 0.19\end{array}$ & $\begin{array}{l}0.89 \\
0.75\end{array}$ & $\begin{array}{l}0.37 \\
0.24\end{array}$ & $\begin{array}{l}0.33 \\
0.20\end{array}$ & $\begin{array}{l}1: 1.1 \\
1.2: 1\end{array}$ & $\begin{array}{l}1: 2.4 \\
1: 3.2\end{array}$ & $\begin{array}{l}1: 2.7 \\
1: 3.8\end{array}$ \\
\hline $\begin{array}{l}\text { 39. } 2.66 \text { kilos } \\
\text { 2. III. } 1929\end{array}$ & 16 & $\mid \begin{array}{ll}\text { R. } & 0.482 \\
\text { L. } & 0.458\end{array}$ & $\begin{array}{l}0.7 \\
0.75\end{array}$ & $\begin{array}{l}0.10 \\
0.065\end{array}$ & $\begin{array}{l}0.062 \\
0.041\end{array}$ & $\begin{array}{l}1.3: 1 \\
2.0: 1\end{array}$ & $\begin{array}{l}1: 6.8 \\
1: 9.2\end{array}$ & $\begin{array}{l}1: 9.1 \\
1: 18.4\end{array}$ \\
\hline $\begin{array}{l}\text { 25. } 2.6 \text { kilos } \\
\text { 12. XI. } 1928\end{array}$ & 17 & $\begin{array}{ll}\text { R. } & 0.33 \\
\text { L. } & 0.39\end{array}$ & $\begin{array}{l}0.8 \\
0.71\end{array}$ & $\begin{array}{l}0.11 \\
0.11\end{array}$ & $\begin{array}{l}0.14 \\
0.12\end{array}$ & $\begin{array}{l}1: 1.8 \\
1: 1.1\end{array}$ & $\begin{array}{l}1: 7.5 \\
1: 6.2\end{array}$ & $\begin{array}{l}1: 5.8 \\
1: 5.8\end{array}$ \\
\hline $\begin{array}{l}\text { 24. } 2.92 \text { kilos } \\
\text { 8. XI. } 1928\end{array}$ & 20 & $\begin{array}{ll}\text { R. } & 0.175 \\
\text { L. } & 0.185\end{array}$ & $\begin{array}{l}1.23 \\
1.3\end{array}$ & $\begin{array}{l}0.11 \\
0.11\end{array}$ & $\begin{array}{l}0.16 \\
0.15\end{array}$ & $\begin{array}{l}1: 1.4 \\
1: 1.4\end{array}$ & $\begin{array}{l}1: 10.1 \\
1: 12.0\end{array}$ & $\begin{array}{l}1: 7.8 \\
1: 8.8^{\circ}\end{array}$ \\
\hline $\begin{array}{l}\text { 23. } 2.74 \text { kilos } \\
\text { 8. XI. } 1928\end{array}$ & 20 & $\mid \begin{array}{ll}\text { R. } & 0.23 \\
\text { L. } & 0.235\end{array}$ & $\begin{array}{l}1.24 \\
1.22\end{array}$ & $\begin{array}{l}0.087 \\
0.085\end{array}$ & $\begin{array}{l}0.099 \\
0.096\end{array}$ & $\begin{array}{l}1: 1.1 \\
1: 1.1\end{array}$ & $\begin{array}{l}1: 14.0 \\
1: 14.0\end{array}$ & $\begin{array}{l}1: 12.7 \\
1: 12.8\end{array}$ \\
\hline $\begin{array}{l}\text { 31. 2.25 kilos } \\
\text { 27. XII. } 1928\end{array}$ & 20 & $\begin{array}{l}\text { R. } 0.24 \\
\text { L. } 0.3\end{array}$ & $\begin{array}{l}0.71 \\
0.76\end{array}$ & $\begin{array}{l}0.31 \\
0.25\end{array}$ & $\begin{array}{l}0.31 \\
0.37\end{array}$ & $\begin{array}{l}1: 1 \\
1: 1.5\end{array}$ & $\begin{array}{l}1: 2.3 \\
1: 3.0\end{array}$ & $\begin{array}{l}1: 2.3 \\
1: 2.0\end{array}$ \\
\hline
\end{tabular}




\begin{tabular}{|c|c|c|c|c|c|c|c|c|}
\hline \multirow{2}{*}{$\begin{array}{l}\text { No. \& weight } \\
\text { of animal } \\
\& \text { date of } \\
\text { experiment }\end{array}$} & \multirow{2}{*}{ 总兽 } & \multirow{2}{*}{ 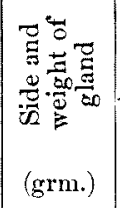 } & \multicolumn{3}{|c|}{$\begin{array}{c}\text { Epinephrine content } \\
\text { (in mgrns. per grm. tissue) } \\
\text { deternined by }\end{array}$} & \multicolumn{3}{|c|}{$\begin{array}{l}\text { Ratios of values } \\
\text { determined by }\end{array}$} \\
\hline & & & Folin & Fye & $\begin{array}{l}\text { Intes- } \\
\text { tine }\end{array}$ & $\begin{array}{l}\text { Eye: } \\
\text { Intes- } \\
\text { tine }\end{array}$ & $\begin{array}{l}\text { Eye : } \\
\text { Folin }\end{array}$ & $\begin{array}{l}\text { Intes- } \\
\text { tine: } \\
\text { Folin }\end{array}$ \\
\hline $\begin{array}{l}\text { 22. } 2.75 \text { kilos } \\
\text { 5. XI. } 1928\end{array}$ & 21 & $\begin{array}{ll}\text { R. } & 0.27 \\
\text { L. } & 0.2 \mathrm{~s}\end{array}$ & $\begin{array}{l}0.97 \\
0.95\end{array}$ & $\begin{array}{l}0.11 \\
0.11\end{array}$ & $\begin{array}{l}0.11 \\
0.11\end{array}$ & $\begin{array}{l}1: 1 \\
1: 1\end{array}$ & $\begin{array}{l}1: 8.7 \\
1: 8.9\end{array}$ & $\begin{array}{l}1: 8.7 \\
1: 8.9\end{array}$ \\
\hline $\begin{array}{l}\text { 26. } 2.86 \text { kilos } \\
\text { 19. XI. } 1928\end{array}$ & $\Sigma 1$ & $\begin{array}{l}\text { R. } 0.24 \\
\text { L. } 0.265\end{array}$ & $\begin{array}{l}0.84 \\
0.75\end{array}$ & $\begin{array}{l}0.12 \\
0.083\end{array}$ & $\begin{array}{l}0.17 \\
0.094\end{array}$ & $\begin{array}{l}1: 1.2 \\
1: 1.1\end{array}$ & $\begin{array}{l}1: 6.7 \\
1: 8.9\end{array}$ & $\begin{array}{l}1: 5.1 \\
1: 8.0\end{array}$ \\
\hline $\begin{array}{l}\text { 28. } 1.66 \text { kilos } \\
\text { 4. XII. } 192 \mathrm{~S}\end{array}$ & 22 & $\begin{array}{l}\text { R. } 0.17 \\
\text { L. } 0.22\end{array}$ & $\begin{array}{l}0.7 \\
0.89\end{array}$ & $\begin{array}{l}0.088 \\
0.14\end{array}$ & $\begin{array}{l}0.073 \\
0.11\end{array}$ & $\begin{array}{l}1.2: 1 \\
1.1: 1\end{array}$ & $\begin{array}{l}1: 8.0 \\
1: 6.6\end{array}$ & $\begin{array}{l}1: 9.6 \\
1: 7.9\end{array}$ \\
\hline $\begin{array}{l}\text { 29. } 2.7 \text { kilos } \\
\text { 11. XII. } 1928\end{array}$ & 22 & $\begin{array}{ll}\text { R. } & 0.17 \\
\text { L. } & 0.18\end{array}$ & $\begin{array}{l}1.0 \\
0.97\end{array}$ & $\begin{array}{l}0.37 \\
0.24\end{array}$ & $\begin{array}{l}0.54 \\
0.28\end{array}$ & $\begin{array}{l}1: 1.5 \\
1: 1.2\end{array}$ & $\begin{array}{l}1: 2.9 \\
1: 4.1\end{array}$ & $\begin{array}{l}1: 1.9 \\
1: 3.5\end{array}$ \\
\hline $\begin{array}{l}\text { 44. } 2.6 \text { kilos } \\
\text { 14. III. } 1929\end{array}$ & 22 & R. $0.2 \$ 1$ & 0.77 & 0.23 & 0.17 & $1.4: 1$ & $1: 3.3$ & $1: 4.6$ \\
\hline $\begin{array}{l}\text { 47. } 2.61 \text { kilos } \\
\text { 11. IV. } 1929\end{array}$ & 22 & L. 0.247 & 0.99 & 0.2 & 0.2 & $1: 1$ & $1: 4.9$ & $1: 4.9$ \\
\hline $\begin{array}{l}\text { 7. } 2.1 \text { kilos } \\
\text { 31. V. } 1928\end{array}$ & 23 & R. 0.26 & 0.55 & 0.11 & 0.096 & $1.2: 1$ & $1: 4.7$ & $1: 5.7$ \\
\hline $\begin{array}{l}\text { 10. } 2.17 \text { kilos } \\
\text { 19. VII. } 1928\end{array}$ & 23 & $\begin{array}{ll}\text { R. } & 0.1 \\
\text { L. } & 0.11\end{array}$ & $\begin{array}{l}1.14 \\
1.08\end{array}$ & $\begin{array}{l}0.17 \\
0.17\end{array}$ & $\begin{array}{l}0.25 \\
0.23\end{array}$ & $\begin{array}{l}1: 1.4 \\
1: 1.4\end{array}$ & $\begin{array}{l}1: 6.6 \\
1: 6.8\end{array}$ & $\begin{array}{l}1: 4.6 \\
1: 4.8\end{array}$ \\
\hline $\begin{array}{l}\text { 40. } 2.29 \text { kilos } \\
\text { 2. III. } 1929\end{array}$ & 23 & L. 0.194 & 1.41 & 0.28 & 0.28 & $1: 1$ & $1: 5.0$ & $1: 5.0$ \\
\hline $\begin{array}{l}9.3 .5 \mathrm{kilos} \\
\text { 11. VII. } 1928\end{array}$ & 24 & $\begin{array}{ll}\text { R. } & 0.25 \\
\text { L. } & 0.21\end{array}$ & $\begin{array}{l}0.41 \\
0.59\end{array}$ & $\begin{array}{l}0.18 \\
0.21\end{array}$ & $\begin{array}{l}0.16 \\
0.09\end{array}$ & $\begin{array}{l}1.1: 1 \\
1.5: 1\end{array}$ & $\begin{array}{l}1: 2.8 \\
1: 2.8\end{array}$ & $\begin{array}{l}1: 2.6 \\
1: 4.2\end{array}$ \\
\hline $\begin{array}{l}\text { 20. } 2.44 \text { kilos } \\
\text { 8. X. } 1928\end{array}$ & 34 & $\begin{array}{l}\text { R. } 0.29 \\
\text { L. } 0.33\end{array}$ & $\begin{array}{l}0.77 \\
0.67\end{array}$ & $\begin{array}{l}0.21 \\
0.18\end{array}$ & $\begin{array}{l}0.21 \\
0.15\end{array}$ & $\begin{array}{l}1: 1 \\
1.2: 1\end{array}$ & $\begin{array}{l}1: 3.7 \\
1: 3.7\end{array}$ & $\begin{array}{l}1: 3.7 \\
1: 4.4\end{array}$ \\
\hline $\begin{array}{l}49.2 .0 \text { kilos } \\
30 . \text { IV. } 1929\end{array}$ & 24 & L. 0.39 & - & 0.15 & 0.15 & $1: 1$ & - & - \\
\hline $\begin{array}{l}\text { 12. } 2.33 \text { kilos } \\
\text { 30. VII. } 1928\end{array}$ & 25 & $\begin{array}{l}\text { R. } 0.27 \\
\text { L. } 0.32\end{array}$ & $\begin{array}{l}0.76 \\
0.82\end{array}$ & $\begin{array}{l}0.13 \\
0.14\end{array}$ & $\begin{array}{l}0.11 \\
0.13\end{array}$ & $\begin{array}{l}1.2: 1 \\
1: 1\end{array}$ & $\begin{array}{l}1: 5.8 \\
1: 5.8\end{array}$ & $\begin{array}{l}1: 6.8 \\
1: 6.2\end{array}$ \\
\hline $\begin{array}{l}\text { 13. 2.I kilos } \\
\text { 30. VII. } 1928\end{array}$ & 25 & $\begin{array}{l}\text { R. } 0.18 \\
\text { L. } 0.2\end{array}$ & $\begin{array}{l}1.34 \\
1.37\end{array}$ & $\begin{array}{l}0.22 \\
0.2\end{array}$ & $\begin{array}{l}0.19 \\
0.12\end{array}$ & $\begin{array}{l}1.1: 1 \\
1.6: 1\end{array}$ & $\begin{array}{l}1: 6.1 \\
1: 6.9\end{array}$ & $\begin{array}{l}1: 69 \\
1: 11.0\end{array}$ \\
\hline $\begin{array}{l}\text { 18. } 2.89 \text { kilos } \\
29 . \text { IX. } 1928\end{array}$ & 25 & L. 0.33 & 0.89 & 0.11 & 0.045 & $2.8: 1$ & $1: 8.4$ & $1: 19.7$ \\
\hline $\begin{array}{l}\text { 45. } 2.59 \text { kilos } \\
\text { 28. III. } 1929\end{array}$ & 25 & L. 0.2 & 1.28 & 0.12 & $0.1 \mathrm{~s}$ & $1: 1.4$ & $1: 9.1$ & $1: 0.5$ \\
\hline $\begin{array}{l}\text { 1. } 1.92 \text { kilos } \\
15 . X .1927\end{array}$ & 26 & $\begin{array}{l}\text { R. } 0.28 \\
\text { L. } 0.28\end{array}$ & $\begin{array}{l}1.02 \\
0.97\end{array}$ & $\begin{array}{l}0.16 \\
0.14\end{array}$ & $\begin{array}{l}0.11 \\
0.067\end{array}$ & $\begin{array}{l}1.5: 1 \\
2.1: 1\end{array}$ & $\begin{array}{l}1: 6.3 \\
1: 6.8\end{array}$ & $\begin{array}{l}1: 9.5 \\
1: 14.5\end{array}$ \\
\hline $\begin{array}{l}\text { 3. } 2.43 \text { kilos } \\
28 . \text { II. } 1928\end{array}$ & 26 & $\begin{array}{l}\text { R. } 0.34 \\
\text { L. } 0.38\end{array}$ & $\begin{array}{l}0.65 \\
0.58\end{array}$ & $\begin{array}{l}0.18 \\
0.16\end{array}$ & $\begin{array}{l}0.15 \\
0.12\end{array}$ & $\begin{array}{l}1.2: 1 \\
1: 1\end{array}$ & $\begin{array}{l}1: 8.7 \\
1: 4.4\end{array}$ & $\begin{array}{l}1: 4.4 \\
1: 4.9\end{array}$ \\
\hline
\end{tabular}




\begin{tabular}{|c|c|c|c|c|c|c|c|c|}
\hline \multirow{2}{*}{$\begin{array}{l}\text { No. \& weight } \\
\text { of animal } \\
\text { \& date of } \\
\text { experiment }\end{array}$} & \multirow{2}{*}{ } & \multirow{2}{*}{ 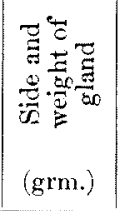 } & \multicolumn{3}{|c|}{$\begin{array}{c}\text { Epinephrine content } \\
\text { (in mgrms. per grm. tissue) } \\
\text { determined by }\end{array}$} & \multicolumn{3}{|c|}{$\begin{array}{l}\text { Ratios of values } \\
\text { determined by }\end{array}$} \\
\hline & & & Folin & Eye & $\begin{array}{l}\text { Intes- } \\
\text { tine }\end{array}$ & $\begin{array}{c}\text { Fye: } \\
\text { Intes- } \\
\text { tine }\end{array}$ & $\begin{array}{l}\text { Eye: } \\
\text { Folin }\end{array}$ & $\begin{array}{l}\text { Intes- } \\
\text { tine: } \\
\text { Folin }\end{array}$ \\
\hline $\begin{array}{l}\text { 4. } 2.45 \text { kilos } \\
28 . \text { II. } 1928\end{array}$ & 26 & $\begin{array}{l}\text { R. } 0.17 \\
\text { L. } 0.18\end{array}$ & $\begin{array}{l}1.2 \\
1.12\end{array}$ & $\begin{array}{l}0.23 \\
0.33\end{array}$ & $\begin{array}{l}0.44 \\
0.26\end{array}$ & $\begin{array}{l}1: 19 \\
1: 1.1\end{array}$ & $\begin{array}{l}1: 5.1 \\
1: 3.4\end{array}$ & $\begin{array}{l}1: 2.7 \\
1: 3.1\end{array}$ \\
\hline $\begin{array}{l}8.2 .12 \mathrm{kilos} \\
\text { 31. V. } 1928\end{array}$ & 26 & $\begin{array}{l}\text { P. } 0.27 \\
\text { L. } 0.27\end{array}$ & $\begin{array}{l}0.38 \\
0.38\end{array}$ & $\begin{array}{l}0.18 \\
0.13\end{array}$ & $\begin{array}{l}0.07 \\
0.07\end{array}$ & $\begin{array}{l}1.9: 1 \\
1.9: 1\end{array}$ & $\begin{array}{l}1: 2.9 \\
1: 2.9\end{array}$ & $\begin{array}{l}1: 5.5 \\
1: 5.5\end{array}$ \\
\hline $\begin{array}{l}\text { 11. } 2.5 \text { kilos } \\
\text { 19. VII. } 1928\end{array}$ & 27 & $\begin{array}{ll}\text { R. } & 0.18 \\
\text { L. } & 0.19\end{array}$ & $\begin{array}{l}0.9 \\
0.85\end{array}$ & $\begin{array}{l}0.17 \\
0.16\end{array}$ & $\begin{array}{l}0.22 \\
0.21\end{array}$ & $\begin{array}{l}1: 1.3 \\
1: 1.8\end{array}$ & $\begin{array}{l}1: 5.4 \\
1: 5.4\end{array}$ & $\begin{array}{l}1: 4.1 \\
1: 4.1\end{array}$ \\
\hline $\begin{array}{l}\text { 15. } 2.36 \text { kilos } \\
\text { 3. IX. } 1928\end{array}$ & 27 & $\begin{array}{l}\text { R. } 0.37 \\
\text { L. } 0.39\end{array}$ & $\begin{array}{l}0.73 \\
0.79\end{array}$ & $\begin{array}{l}0.27 \\
0.28\end{array}$ & $\begin{array}{l}0.3 \\
0.31\end{array}$ & $\begin{array}{l}1: 1.1 \\
1: 1.1\end{array}$ & $\begin{array}{l}1: 2.7 \\
1: 2.7\end{array}$ & $\begin{array}{l}1: 2.5 \\
1: 2.5\end{array}$ \\
\hline $\begin{array}{l}\text { 16. } 2.55 \text { kilos } \\
\text { 10. IX. } 1928\end{array}$ & 27 & $\begin{array}{l}\text { R. } 0.25 \\
\text { L. } 0.26\end{array}$ & $\begin{array}{l}0.56 \\
0.55\end{array}$ & $\begin{array}{l}0.2 \\
0.23\end{array}$ & $\begin{array}{l}0.16 \\
0.15\end{array}$ & $\begin{array}{l}1.2: 1 \\
1.5: 1\end{array}$ & $\begin{array}{l}1: 2.8 \\
1: 2.4\end{array}$ & $\begin{array}{l}1: 3.5 \\
1: 3.6\end{array}$ \\
\hline $\begin{array}{l}21.3 .06 \text { kilos } \\
22 . \times .1928\end{array}$ & 27 & $\begin{array}{l}\text { R. } 0.45 \\
\text { I. } 0.46\end{array}$ & $\begin{array}{l}1.08 \\
0.95\end{array}$ & $\begin{array}{l}0.15 \\
0.13\end{array}$ & $\begin{array}{l}0.11 \\
0.06\end{array}$ & $\begin{array}{l}1.4: 1 \\
2.0: 1\end{array}$ & $\begin{array}{l}1: 6.9 \\
1: 7.3\end{array}$ & $\begin{array}{l}1: 9.7 \\
1: 14.5\end{array}$ \\
\hline $\begin{array}{l}\text { 17. } 3.1 \text { kilos } \\
24 . \text { IX. } 1928\end{array}$ & 28 & $\begin{array}{ll}\text { R. } & 0.20 \\
\text { L. } & 0.211\end{array}$ & $\begin{array}{l}1.13 \\
0.95\end{array}$ & $\begin{array}{l}0.12 \\
0.12\end{array}$ & $\begin{array}{l}0.11 \\
0.08\end{array}$ & $\begin{array}{l}1.1: 1 \\
1.4: 1\end{array}$ & $\begin{array}{l}1: 9.1 \\
1: 8.0\end{array}$ & $\begin{array}{l}1: 10.1 \\
1: 11.4\end{array}$ \\
\hline $\begin{array}{l}\text { 50. } 1.97 \text { kilos } \\
\text { 9. V. } 1929\end{array}$ & 28 & R. 0.25 & 1.8 & 0.1 & 0.12 & $1: 1.2$ & $1: 13.0$ & $1: 10.8$ \\
\hline $\begin{array}{l}\text { 5. } 2.69 \text { kilos } \\
24 . \text { IV. } 1928\end{array}$ & 29 & $\begin{array}{ll}\text { R. } & 0.225 \\
\text { L. } & 0.22\end{array}$ & $\begin{array}{l}0.94 \\
1.03\end{array}$ & $\begin{array}{l}0.13 \\
0.14\end{array}$ & $\begin{array}{l}0.27 \\
0.23\end{array}$ & $\begin{array}{l}1: 2.1 \\
1: 1.7\end{array}$ & $\begin{array}{l}1: 7.1 \\
1: 7.6\end{array}$ & $\begin{array}{l}1: 3.4 \\
1: 4.5\end{array}$ \\
\hline $\begin{array}{l}\text { 30. } 2.47 \text { kilos } \\
\text { 18. XII. } 1928\end{array}$ & 29 & $\begin{array}{l}\text { R. } 0.185 \\
\text { L. } 0.22\end{array}$ & $\begin{array}{l}1.12 \\
1.03\end{array}$ & $\begin{array}{l}0.13 \\
0.11\end{array}$ & $\begin{array}{l}0.12 \\
0.1\end{array}$ & $\begin{array}{l}1.1: 1 \\
1.1: 1\end{array}$ & $\begin{array}{l}1: 8.3 \\
1: 9.0\end{array}$ & $\begin{array}{l}1: 9.5 \\
1: 11.1\end{array}$ \\
\hline $\begin{array}{l}\text { 19. } 2.86 \text { kilos } \\
29.1 \times .1928\end{array}$ & 30 & $\begin{array}{l}\text { R. } 0.16 \\
\text { L. } 0.19\end{array}$ & $\begin{array}{l}0.94 \\
0.82\end{array}$ & $\begin{array}{l}0.22 \\
0.21\end{array}$ & $\begin{array}{l}0.12 \\
0.16\end{array}$ & $\begin{array}{l}1.7: 1 \\
1.3: 1\end{array}$ & $\begin{array}{l}1: 4.3 \\
1: 3.9\end{array}$ & $\begin{array}{l}1: 7.5 \\
1: 5.2\end{array}$ \\
\hline $\begin{array}{l}\text { 27. } 2.6 \text { kilos } \\
\text { 29. XI. } 1928\end{array}$ & 30 & $\begin{array}{l}\text { R. } 0.27 \\
\text { L. } 0.28\end{array}$ & $\begin{array}{l}0.86 \\
0.89\end{array}$ & $\begin{array}{l}0.15 \\
0.21\end{array}$ & $\begin{array}{l}0.17 \\
0.21\end{array}$ & $\begin{array}{l}1: 1.1 \\
1: 1\end{array}$ & $\begin{array}{l}1: 5.8 \\
1: 4.2\end{array}$ & $\begin{array}{l}1: 5.2 \\
1: 4.2\end{array}$ \\
\hline $\begin{array}{l}\text { 33. 2.69 kilos } \\
\text { 19. I. } 1929\end{array}$ & $\begin{array}{c}\text { ca. } 8 \\
\text { hours } \\
\text { after }\end{array}$ & $\begin{array}{l}\text { R. } 0.356 \\
\text { L. } 0.365\end{array}$ & $\begin{array}{l}0.69 \\
0.65\end{array}$ & $\begin{array}{l}0.15 \\
0.14\end{array}$ & $\begin{array}{l}0.15 \\
0.14\end{array}$ & $\begin{array}{l}1: 1 \\
1: 1\end{array}$ & $\begin{array}{l}1: 4.4 \\
1: 4.7\end{array}$ & $\begin{array}{l}1: 4.4 \\
1: 4.7\end{array}$ \\
\hline $\begin{array}{l}\text { 32. } 2.55 \text { kilos } \\
\text { 14. I. } 1929\end{array}$ & $\begin{array}{c}2 \text { days } \\
\text { after }\end{array}$ & $\begin{array}{l}\text { R. } 0.14 \\
\text { L. } 0.195\end{array}$ & $\begin{array}{l}0.87 \\
0.75\end{array}$ & $\begin{array}{l}0.26 \\
0.19\end{array}$ & $\begin{array}{l}0.3 \\
0.19\end{array}$ & $\begin{array}{l}1: 1.1 \\
1: 1\end{array}$ & $\begin{array}{l}1: 3.3 \\
1: 3.9\end{array}$ & $\begin{array}{l}1: 2.9 \\
1: 3.9\end{array}$ \\
\hline
\end{tabular}

The surplus of epinephrine-Eye over epinephrine-Intestine was also large in the period from the 24 th day of pregnancy to the $27 \mathrm{th}$, when the reverse ratio frequently occurred.

By comparing these results with those of the parallel estimations of the cava pocket blood, it may be pointed out that in the cases where the content of epinephrine-Intestine was greater than that of epinephrine-Eye, 
the latter was found rather in a greater amount than the epinephrine-Intestine in the gland excised after the collection of the samples. This reciprocal relation between the blood and the extract can be detected by comparing not only the averages in various stages of pregnancy in both tables, but the ratios for the blood and the extract in one and the same animal. Though the relation was not invariably seen in individual cases, in the majority of cases the tendency was clearly established. This is apparently paradoxical, but may be reasonably explained in a such way that the epinephrine-Intestine is particularly abundantly discharged from the suprarenal body into the suprarenal vein in a certain advanced stadium of pregnancy, whereby that substance is much exhausted in the gland. It is beyond dispute that the epinephrine discharge is accelerated remarkably in such experimental conditions as in the present research in comparison with the natural, quiet life of the animal.

The other ratios for the gland extracts were as follows: Eye : Folin $1: 1.5$ to $1: 16.6$, on an a verage $1: 5.8$, Intestine : Folin $1: 1.4$ to $1: 18.0$, mean $1: 6.2$. In general the epinephrine-Folin was found incomparably copious in the glands of the pregnant rabbits. That the extract of the cortex of the rabbit suprarenal capsule contains the Folin positive substance in abundance but clicits no sympathomimetic action was witnessed in this laboratory. The hypertrophy of the suprarenal cortex during pregnancy is generally accepted.

The content (mgrm. per grm. tissue) of the epinephrine of the suprarenals of non-pregnant rabbits, left after collecting the suprarenal vein blood by means of the cava-pocket was previously ${ }^{1}$ estimated, that in the present investigation are as follows:

\begin{tabular}{|c|c|c|c|c|c|c|c|c|c|c|c|c|c|}
\hline & & & & & & & & $\underset{I_{n}}{\text { Epin }}$ & $\begin{array}{l}\text { hrine- } \\
\text { tine }\end{array}$ & Eping & arine- & $\mathrm{E}_{\mathrm{p}} \mathrm{i}_{\mathrm{I}}$ & $\begin{array}{l}\text { hrine. } \\
\text { in }\end{array}$ \\
\hline Non-pregn & ant & & & (18: & nim & $\mathrm{lgla}$ & ads) & 0.26 & agrm. & 0.22 & grm. & 0.81 & grin. \\
\hline $4 t h-10 t h$ & $\mathrm{day}$ & of & gnancy & $(6)$ & nim & al $9 \mathrm{~g}$ & lands) & 0.36 & " & 0.31 & $"$ & 1.0 & $"$ \\
\hline 12 th-20th & " & , & $"$ & $(9$ & ", & 17 & ") & 0.18 & $"$ & 0.16 & $n$ & 1.0 & " \\
\hline 21 st - 25th & $"$ & $"$ & " & $(26$ & $"$ & 44 & $"$ & 0.17 & $"$ & 0.18 & , & 0.86 & $"$ \\
\hline 29 th & $"$ & $\eta$ & " & $(2$ & , & 4 & ") & 0.18 & , & 0.13 & " & 1.0 & , \\
\hline 30 th & $"$ & & $"$ & 2 & " & 4 & ", & 0.16 & $"$ & 0.2 & $n$ & 0.88 & $"$ \\
\hline Puerperal & $\operatorname{tag}$ & & & $(2$ & , & 4 & , & 0.19 & $"$ & 0.18 & $"$ & 0.74 & $n$ \\
\hline
\end{tabular}

The epinephrine deterninable by the rabbit intestine strip and by the denervated eye of the cat was found specially small in the suprarenals left after collecting the cava pocket blood in the advanced stage of the pregnancy compared with the non-pregnant rabbits, and in the beginning of the pregnancy it was found in greater amount. The Folin positive substance was found increased during the pregnancy.

4) Sugawara, Tohoku J. Exp. Med., 1928, II, 420-421; Watanabé and Sato, Ibid., 1928, II, 483. 


\section{Summary.}

The cava pocket blood sample of rabbits was taken at various stages of pregnancy, and was tested against adrenalin chloride of the Sankyo Co. by means of the rabbit intestine strip method and the paradoxical eye reaction in parallel. From the suprarenals left behind in the cava pocket experiment the acid extract was prepared and also tested by both the biological methods and the colorimetrical method of Folin, Cannon and Denis.

While the suprarenal vein blood of the non-pregnant rabbit involves a greater ability of dilating the denervated pupil than of inhibiting the rabbit intestine movement, the pregnancy alters the ability, and this becomes remarkable with the advancement of the same, the ratio of the value obtainable by the denervated pupil to that by the rabbit intestine being recorded as $1: 1.5$ in this stage on an average against the average of $2.2: 1$ for the non-pregnant rabbit. The ratio becomes reverse.

In respect of the ratio of the values obtained by both biological methods, the suprarenal vein blood and the extract of the suprarenal gland left behind after the cava pocket experiment show a reciprocal relation; it is readily intelligible when it is taken into consideration that the over-secretion of a substance can act to diminish its storage in the gland. 